\title{
JOINT GRAPH COLORING AND ANT COLONY OPTIMIZATION BASED SUB-CHANNEL ALLOCATION ALGORITHMS FOR DOWNLINK LTE-A HETNETS
}

\author{
by \\ Richa Siddavaatam \\ B.Eng., Pune University, India, 2005
}

A Thesis

Presented to the School of Graduate Studies at Ryerson University

in partial fulfillment of the requirements for the degree of

Master of Applied Science

in the Program of Electrical and Computer Engineering

Toronto, Ontario, Canada, August 2012

(C)Richa Siddavaatam 2012 


\section{Author's Declaration}

I hereby declare that I am the sole author of this thesis. This is a true copy of the thesis, including any required final revisions, as accepted by my examiners.

I authorize Ryerson University to lend this thesis to other institutions or individuals for the purpose of scholarly research.

Author's Signature:

I further authorize Ryerson University to reproduce this thesis by photocopying or by other means, in total or in part, at the request of other institutions or individuals for the purpose of scholarly research.

I understand that my thesis may be made electronically available to the public.

Author's Signature: 


\section{Abstract \\ Graph Coloring and Ant Colony Optimization based Sub-channel Allocation Algorithms for LTE-A HetNets}

(C)Richa Siddavaatam, 2012

Master of Applied Science

Electrical and Computer Engineering

Ryerson University

LTE-Advanced is a promising technology which supports much higher peak rates, higher throughput and coverage, and lower latencies, resulting in a better user experience. For extended indoor coverage LTE-A supports two-tier network composed of conventional macrocellular networks and femtocell networks known as HetNets. As the femtocell shares the same frequency band with underlying macrocell, the cross tier interference needs to be mitigated. The inter-femtocell and cross tier interference near femtocell boundary may result in unwarranted degradation of system performance. In LTE-A, as an OFDM based technology, we consider the use of prudent PRB (Physical Resource Block) allocation to mitigate downlink inter-femtocell interference as well as cross tier interference in the mentioned environment for this small HetNet. Allocation of PRBs to network users is formulated as a graph coloring problem. Based on this interpretation and initial sensitivity study, we propose dynamic resource allocation algorithms namely greedy allocation (GA), SINR based allocation (SINRA) and ant colony allocation (ACA). From the simulation results and its analysis we can conclude that, ACA algorithm outperforms rest of the considered algorithms while GA and SINRA provide performance with the significant improvement over random allocation (RA) algorithm as far as performance parameters such as averaged SINR experienced by a network user, outage probability and number of PRBs needed are concerned. 


\section{Acknowledgement}

I would like to express my sincere gratitude to many people who made this thesis possible.

I have been greatly indebted in the preparation of this thesis to my supervisor, Dr.Alagan Anapalagan. Thanks to his engaging personality and enthusiasm and moreover his great efforts to clarify concepts and simplify terms, helped to make communication systems fun for me. Throughout the period of my masters studies he provided encouragement, sound advice, exemplary teaching and a whole lot of nice ideas. I would have been lost without him.

I would like to acknowledge the Department of Electrical and Computer Engineering and the School of Graduate Studies at Ryerson University for their support in terms of financial aid, and work experience as a graduate assistant.

I would also like to thank my defense committee members Dr.Olivia Das, Dr.Isaac Woungang, Dr.Xiao-Ping Zhang and Dr.Alagan Anapalagan for taking the time and effort to review my work and provide me with their insightful comments.

I can never find the words to thank my husband Prathap, who was amazingly patient and understanding. Without his support and love, I would not be where I am today. He not only believed in me and was always supportive but he also offered me his advice and his valuable help.

My special thanks goes to my parents, Mr.Daji Gawade and Ms.Arati Gawade who are always a great source of love and support. Last but not least, I would like to thank my bundle of joy Aarav who has been my stress reliever and reason to be happy.

Richa 


\section{Contents}

1 Introduction 1

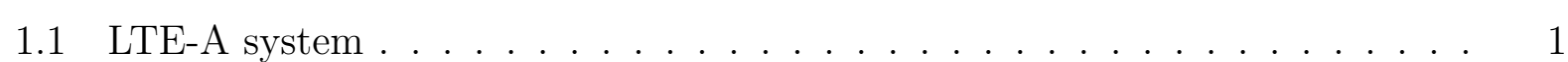

1.2 Interference management in LTE-A HetNet . . . . . . . . . . . . . . . . . 3

1.3 Evolutionary algorithms for channel allocation . . . . . . . . . . . . . . 4

1.4 Thesis organization . . . . . . . . . . . . . . . . . . . 4

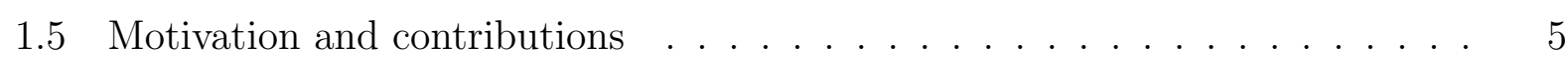

2 Literature Survey: Interference management in cellular network $\quad 7$

3 Model and Sensitivity Analysis $\quad 16$

3.1 Network model . . . . . . . . . . . . . . . . . . . . . . . . . . 16

3.1 .1 LTE-A phvsical laver . . . . . . . . . . . . . . . . . . 16

3.1 .2 Channel model . . . . . . . . . . . . . . . . . . . . . 19

3.1 .3 Svstem parameters ........................ 20

3.1.4 Determination of cell association . . . . . . . . . . . . 20

3.2 Mathematical model of PRB assignment problem . . . . . . . . . . . . . 21

3.3 PRB allocation as graph coloring problem . . . . . . . . . . . . . . . . . . 22

3.4 Sensitivity studv . . . . . . . . . . . . . . . . . . . . . . . . . 24

3.4 .1 Interference graph . . . . . . . . . . . . . . . . . . . . . . 24

3.4 .2 Channel availability . . . . . . . . . . . . . . . . . . 27

3.4 .3 Cell association . . . . . . . . . . . . . . . . . 30 
4 Proposed Subchannel Allocation Algorithms

4.1 Ant colonv optimization . . . . . . . . . . . . . . . 33

4.1 .1 Biological inspiration . . . . . . . . . . . . . . . . . 34

4.1.2 Ant colonv optimization metaheuristid . . . . . . . . . . . . . 34

4.2 ACO algorithm for PRB allocation . . . . . . . . . . . . . . 35

4.2.1 Definition of trail factor . . . . . . . . . . . . . . . 38

4.2 .2 Highlights of the algorithm . . . . . . . . . . . . . . . . . . 40

4.3 Greedv algorithm for graph coloring . . . . . . . . . . . . . . . . . . . . 43

4.4 Greedy allocation and SINR based allocation for PRB allocation problem . . 43

5 Performance of the Proposed Algorithms 48

5.1 Convergence analysis of ant colonv allocation algorithm . . . . . . . . . . . 48

5.2 Comparative study of different algorithms . . . . . . . . . . . . . . . . 49

5.2 .1 Performance with change in SINR threshold . . . . . . . . . . . . 50

5.2.2 Performance with change transmit power of FAPs . . . . . . . . . 56

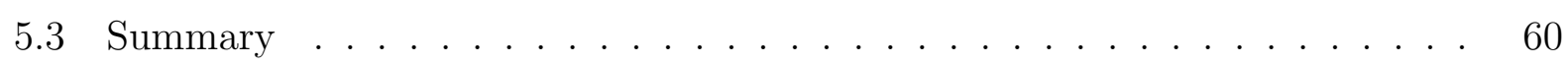

6 Conclusion and Future work $\quad 62$

6.1 Conclusion . . . . . . . . . . . . . . . . . . . 62

6.2 Future work . . . . . . . . . . . . . . . . . . . . . . 63 


\section{List of Figures}

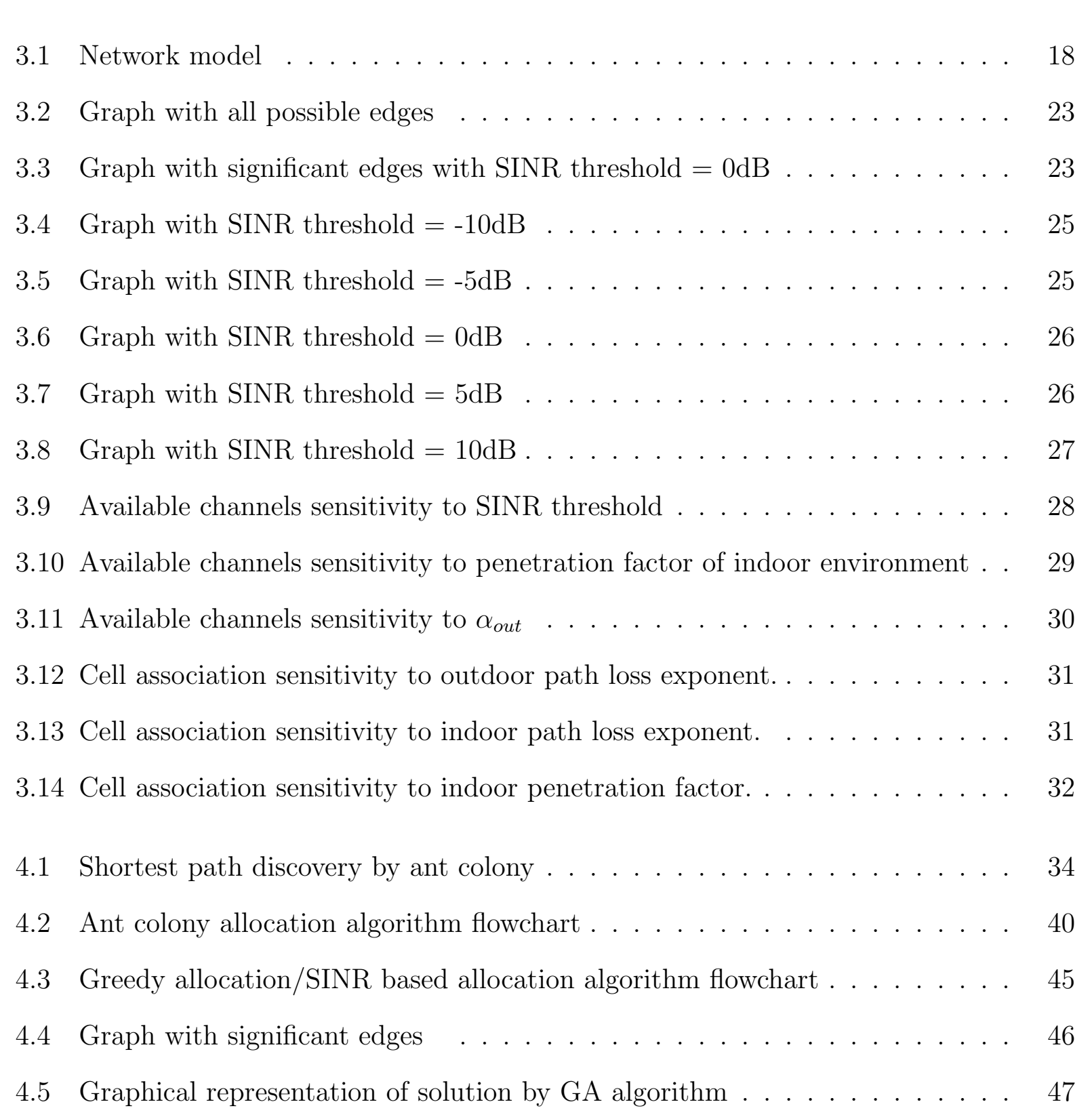




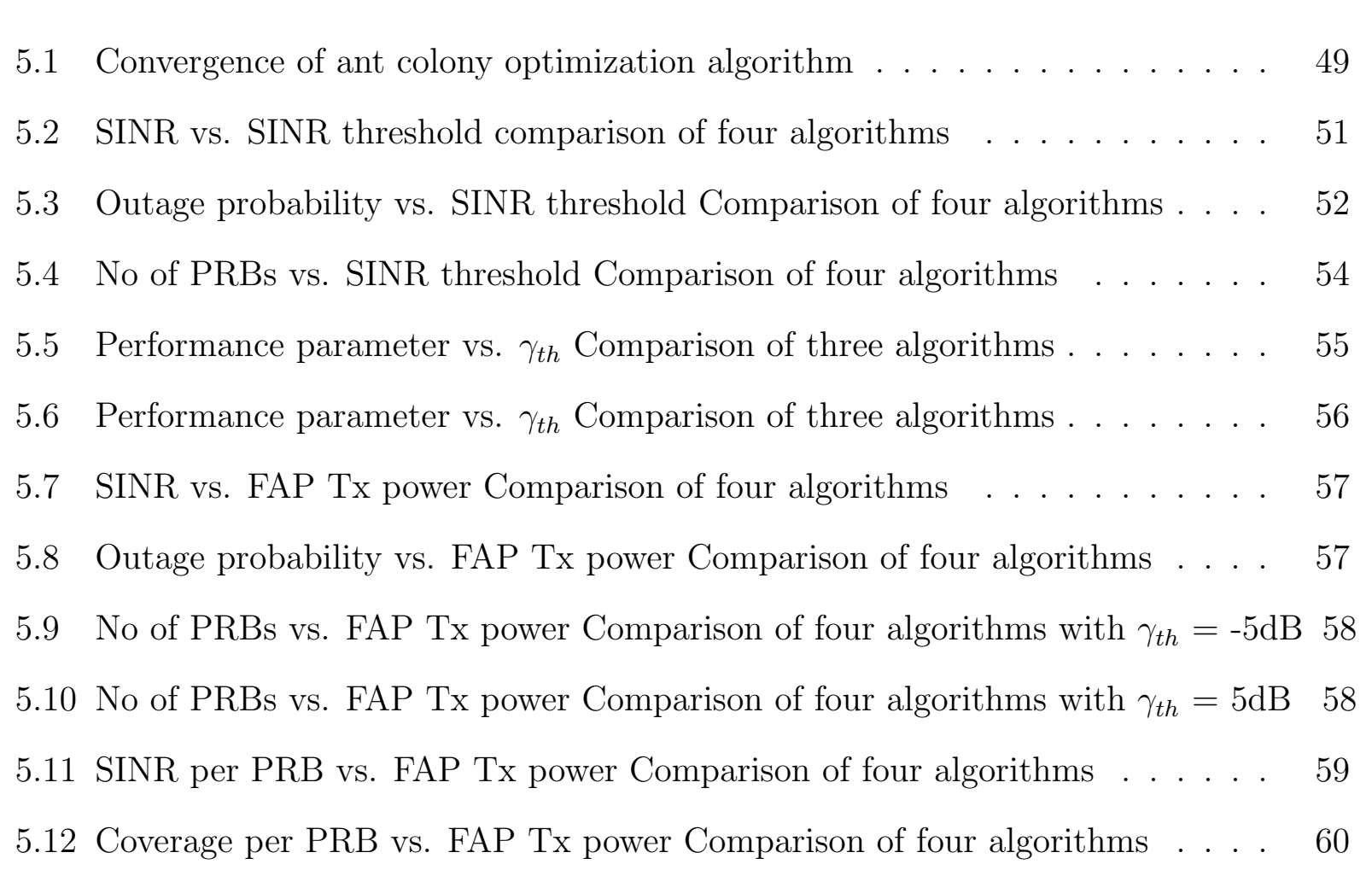




\section{List of Tables}

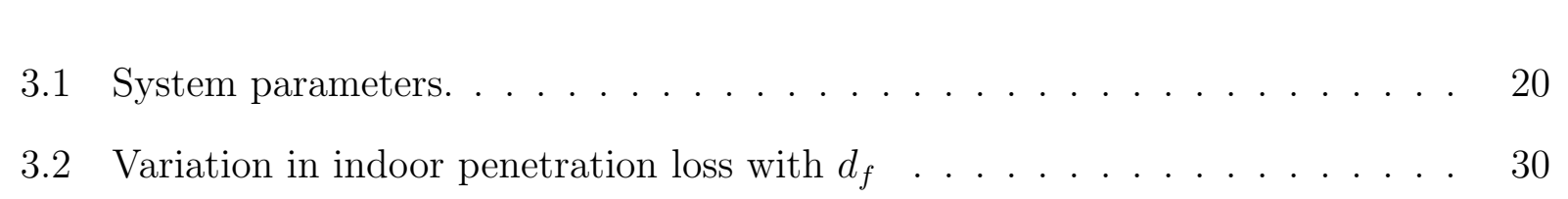

4.1 Description of variables used $\ldots \ldots \ldots \ldots$

4.2 Ant colony allocation parameters . . . . . . . . . . . . . . . 41

5.1 Summary of performance analysis of proposed algorithm . . . . . . . . . 61 


\section{List of Abbreviations}

\begin{tabular}{ll}
$1 \mathrm{G}$ & First Generation \\
$2 \mathrm{G}$ & Second Generation \\
$3 \mathrm{G}$ & Third Generation \\
$4 \mathrm{G}$ & Fourth Generation \\
PRB & Physical resource block \\
LTE & Long Term Evolution \\
LTE-A & Long Term Evolution-Advanced \\
CDMA & Code Division Multiple Access \\
BPSK & Binary Phase Shift Keying \\
BS & Base Station \\
UE & User Equipment \\
FAP & Femtocell Access Point \\
GCP & Graph Coloring Problem \\
OFDMA & Orthogonal Frequency Division Multiple Access \\
SC-OFDMA & Single Carrier Orthogonal Frequency Division Multiple Access \\
HetNets & Heterogeneous Networks \\
FFR & Fractional Frequency Reuse \\
QoS & Quality of Service \\
RSSI & Received Signal Strength Indicator \\
SCN & Small Cell Network \\
ACO & Ant Colony Optimization \\
GA & Greedy Allocation \\
\hline
\end{tabular}




$\begin{array}{ll}\text { RA } & \text { Random Allocation } \\ \text { ACA } & \text { Ant Colony Allocation } \\ \text { SINRA } & \text { SINR based Allocation } \\ \text { SINR } & \text { Signal to Interference and Noise Ratio } \\ \text { SNR } & \text { Signal to Noise Ratio } \\ \text { CAP } & \text { Channel Assignment Problem } \\ \text { SA } & \text { Simulation Annealing } \\ \text { RSRP } & \text { Reference Signal Received Power } \\ \text { PAPR } & \text { Peak to Average Power Ratio } \\ \text { SI } & \text { Swarm Intelligence } \\ \text { IMT-A } & \text { International Mobile Telecommunications-Advanced } \\ \text { eNB } & \text { Evolved Node B } \\ \text { DAS } & \text { Distributed Antenna Systems } \\ \text { ICIC } & \text { Intercell Interference Coordination } \\ \text { RF } & \text { Radio Frequency } \\ \text { EA } & \text { Evolutionary Algorithm }\end{array}$




\section{Chapter 1}

\section{Introduction}

Wireless communication is the fastest growing element of telecommunication industry. A cellular system has experienced exponential growth over last decades and has become inseparable part of modern society. They are expected to grow continuously and face stringent challenges as far as performance improvement at low cost is concerned. The cellular communication standard has rapidly evolved from 1st generation with analog radio signals to digital signals based 2nd generation $(2 \mathrm{G})$ standard. 2G was primarily voice only but does provide higher bandwidth for better voice quality and limited data services. The 3rd generation $(3 \mathrm{G})$ was the next milestone featuring high data rate up to 2 Mbps which supported data applications including multimedia and internet services. Now, the focus is on 4th generation (4G) standard.

\subsection{LTE-A system}

Long Term Evolution (LTE) is a 4th generation standard for wireless data communication which has evolved from the GSM/UMTS standards. LTE-A is an evolved version of LTE release 8 and is expected to satisfy the IMT-A system requirements for high peak data rate (downlink 3Gbps/uplink 1.5 Gbps), spectral efficiency (30 bps/Hz), improved performance at cell edges (min $2.40 \mathrm{bps} / \mathrm{Hz} / \mathrm{cell})$ [1]. In order to meet these requirements, the techniques 
such as carrier aggregation, uplink and downlink spatial multiplexing, downlink intracell coordinated multipoint (CoMP) transmission and reception have been incorporated in LTEA standard. In addition to these technologies, the desired spectral efficiency and coverage enhancement can be achieved by deploying a two tier heterogeneous network (HetNet), which is comprised of low power nodes distributed across macrocellular network. A variety of low power nodes exists which suits well for various environment such as micro-cells for hot-spots, pico-cells for enterprise, femtocells for home environment and, relay nodes are suitable for cell edges.

LTE-A is expected to satisfy IMT-Advanced requirements in terms of capacity, data rates and low-cost deployment. This includes the possibility for peak data rates up to 1 Gbps in the downlink and 500 Mbps in the uplink. The link performance of current cellular systems such as LTE is already quite close to the Shannon limit [2]. From link-budget perspective, the very high data rates targeted by LTE-Advanced require a higher SINR performance. Hence, it is imperative to innovate the technologis which would improve SINR performance.

Heterogeneous network is a two-tier network comprised of a lower tier of macrocell and the higher tier of multiple low power nodes. The low power nodes can be micro eNBs, pico eNBs, home eNBs (femtocells), relays and distributed antenna systems (DAS). These short range nodes enhance the network performance at low cost and low power. Since these low power nodes operate on the same spectrum as macrocell network, the role of intercell interference coordination (ICIC) is critical in obtaining good performance. We consider a two-tier HetNet consisting of multiple femtocells and underlaying macrocell. Femtocells are deployed and maintained by end users in plug and play manner. Their interference environment is more complicated than the traditional macro-cell environment. Since the quantity and the position of femtocells vary randomly, interference mitigation needs to be dealt with specifically designed techniques. Due to semi-statically varying network layout, dynamic resource allocation is a feasible solution for femtocell based network.

A significant performance leap is expected from HetNets, which brings the network closer 
to the user through low-power, low-range nodes such as pico and femto-cells. LTE Advanced needs adaptive interference management techniques to further improve the capacity and coverage of these HetNets. In this thesis, we propose dynamic channel allocation algorithms namely greedy allocation, SINR based allocation and ant colony allocation for LTE-A downlink transmission in two-tier network environment.

\subsection{Interference management in LTE-A HetNet}

In our thesis, we consider a two tier heterogeneous network consisting of multiple femtocells sometimes referred to as small cell networks (SCN) [3] and underlying macrocell. Femtocell base stations are low cost, low power, short range base stations deployed and maintained by end-users in order to achieve better indoor coverage and higher data rates at lower cost. They operate in the same frequency spectrum as macrocell and connect to macrocell network through backhaul connections. Since femtocell users communicate with short range base stations, they are expected to transmit at lower power which leads to energy conservation and thereby prolonging longevity of battery life. The main purpose of femtocell deployment is to extend indoor coverage. Moreover, the appropriate placement of femtocell access point (FAP) offloads the traffic load from macrocell to femtocells improving network capacity and quality of service (QoS). Although HetNet is a promising technology, it presents many technological challenges such as interference mitigation, network security, health hazards from increased $\mathrm{RF}$ radiation etc. The foremost challenge is the mitigation of intra-tier interference of femtocell tier and inter-tier interference as femtocells operate on the same spectrum as macrocell. LTE-A is an OFDMA based system; transmission is scheduled by physical resourse block (PRB) which is a set of 12 consecutive sub-carriers. An proper allocation of available PRBs would be a potential solution for the interference mitigation problem. 


\subsection{Evolutionary algorithms for channel allocation}

Evolutionary algorithms (EA) are a class of probabilistic search techniques based on the selection mechanism adopted by natural systems [4]. They operate as follows: the potential solutions are called individuals which form a population which undergoes a transformation. The better solutions according to the selection criterion called 'fitness function', are selected to constitute a next generation. When a pre-defined condition is satisfied after a number of generations, the algorithm terminates and the fittest solution of the last generation is presented as the solution to the problem under consideration. Due to the existence of certain stringent constraints on channel assignment problem of cellular network, the deterministic channel allocation methods have been proven to be time consuming and complex [5]. The foremost constraint on the channel allocation problem in modern cellular network is minimization of co-channel interference. This constraint prohibits the users which are located within certain distance from being assigned the same channel. Many heuristics have been suggested for this problem which includes simulated annealing, neural networks and genetic algorithms. These methods would be reviewed in the following chapter. Swarm intelligence is one of the most promising evolutionary algorithms for this particular problem. In this thesis, we propose channel allocation algorithm based on ant colony optimization which is a type of swarm intelligence.

\subsection{Thesis organization}

With the introduction of LTE-A two-tier network and an evolutionary algorithm which can be a efficient solution to the identified interference mitigation problem through sub-channel allocation, in chapter 2, we present a literature review regarding the techniques that have been applied to similar problems in the contemporary literature.

In chapter 3, we define the two-tier network model and its parameters which we would be utilising for simulation purposes for the evaluation of the proposed algorithms. The network 
model includes description of LTE-A downlink, indoor and outdoor channel models, system parameters and mathematical model of sub-channel assignment problem. We also describe the cell association method and conduct sensitivity study of the network model in order to verify its reliability. As well the interference graph representation of PRB allocation problem is detailed in this chapter.

Chapter 4 introduces the readers to ant colony optimization technique along with the biological inspiration behind it and provides ant colony optimization metaheuristics. We present the procedural details of the proposed algorithms namely greedy allocation (GA), SINR based allocation (SINRA) and ant colony allocation (ACA). We also describe random allocation (RA) algorithm which would be used for comparison purposes during performance analysis of the proposed algorithms. The some preliminary results of greedy allocation algorithm are also given in this chapter.

In chapter 5, we conduct performance analysis of the proposed algorithms and also perform a comparative study of these algorithms with each other and with random allocation (RA) method. ACA being an iterative algorithm, we would conduct convergence analysis in order to determine the appropriate number of iterations it needs to execute for optimum results. Based on the performance analysis results, we conclude the thesis in chapter 6 and provide a brief discussion regarding the future research.

\subsection{Motivation and contributions}

The interference mitigation and management have been identified as a predominant challenge in LTE-A HetNets. Since the femtocell tier of HetNet operates on the same spectrum as macro-cell tier, the prudent spectrum sharing among femtocell and macrocell users would be an efficient solution for interference mitigation problem. We propose dynamic channel allocation algorithms which would be best suited for dynamically-varying wireless environment of LTE-A HetNet. The contributions of this thesis can be summarized as follows: 
- Defined and evaluated the performance of a typical two-tier LTE-A network model.

- Proposed 'Ant colony allocation' an evolutionary sub-channel allocation algorithm based on ant colony optimization technique.

- Proposed 'Greedy algorithm' for sub-channel allocation in the downlink of LTE-A HetNet which is based on greedy graph coloring algorithm.

- Proposed 'SINR based allocation' for sub-channel allocation in the downlink of LTE-A HetNet which is based on greedy graph coloring algorithm.

- Conducted comparative performance analysis of the proposed algorithms with that of random allocation algorithm. 


\section{Chapter 2}

\section{Literature Survey: Interference management in cellular network}

The channel assignment problem is considered NP-complete and is closely related to graph coloring problem [6]. Determination of an optimal solution is infeasible due to exponentially growing computation time. Various algorithmic techniques such as simulated annealing, genetic algorithms, neural networks, game theory, and adaptive local search have been applied in the past in order to solve this problem. Authors of [7] have presented the dynamic channel assignment problem as a combinatorial optimization problem and solved it using modified Hopfield neural network. The proposed algorithm outperforms other neural networks such as winner-take-all neural network [8] and feed-forward network as far as call blocking probability is concerned. Another neural network approach suggested in [9] combines sequential heuristic algorithms into a parallel neural network algorithm. The algorithm operates in three stages; 1) the regular interval assignment stage: 2) the greedy assignment stage and 3) the neural-network assignment stage. In the first stage, the calls in a cell which determine the lower bound on the total number of channels are assigned channels at a regular interval. In the second stage, the calls in a cell with the highest degree and those in adjacent cells are assigned channels by a greedy heuristic method. In the third stage, the calls in 
the remaining cells are assigned channels by a binary neural network. When applied to the benchmark problems, this algorithm could achieve a good solution in reasonable time [9]. A two stage channel assignment algorithm based on self-organizing mechanism of swarm intelligence and a transiently chaotic neural network has been introduced [10]. Self-inhabiting effects of chaotic neuron is applied in order to prevent the process from getting trapped into local minima. When applied to the benchmark problems, the algorithm results in improved performance in comparable time to that of other heuristics.

The approach in [11] combines simulated annealing or neural network techniques in order to solve the channel assignment problem. Genetic algorithm is used to generate call ordering list and channel exhaustive allocation is implemented for channel allocation. The iterative procedure reduces the search space to high quality solutions leading to faster convergence of the process. With application to benchmark problems this algorithm outperforms other considered algorithms. Further the authors in [11] claims that the possibility of parallelization execution time could be reduced significantly. The authors in [12] proposes a dynamic channel assignment algorithm consisting of three stages: 1) a determine-lower-bound cell regular interval assignment stage 2) a greedy region assignment stage and 3) a genetic algorithm assignment stage. The performance is verified through application to Philadelphia problem [13] which achieves lower bound solutions and execution time is lower than the approaches using neural-networks and simulated annealing. The authors of [14] suggest application of genetic algorithm to a small subset of the network with homogeneous demands and then extend the solution to entire network. Their algorithm exhibits improved performance in terms of optimality of bandwidth and computation time compared to most of the techniques presented in earlier literature.

From a mathematical point of view, the CAP (channel assignment problem) can be considered as combinatorial optimization problem with constraints. Being a common approach to solve combinatorial optimization problem, simulated annealing has been applied to solve CAP. In simulated annealing, the cost function quantifies channel separation constraints and 
channel demands, which reaches zero when all constraints are satisfied. Two independent approaches differing in models and neighborhood structure are proposed in [15] and [16].

Many ACO algorithms have been proposed for suboptimal solution of channel allocation problem. But each of the mentioned algorithms varies in problem formulation and parameter definition for ACO framework. Authors in [17] implement ANTS (Approximate Nondeterministic Tree Search) - a member of ACO algorithm family for solving minimum span channel assignment problem. Instead of defining trail levels on moves from a state to another, authors have defined them on transmitter channel pair. It gives an indication of how proficient it has been in the past to assign a channel to a transmitter. As per the technique presented in [18] each base station of multi-cell network applies ant colony optimization (ACO) to choose available sub-channels dynamically, which contributes to reduce co-channel interference. As per pheromone associated with channel capacity, the probability of choosing sub-channel with better capability is higher. The algorithm could achieve fairly good spectral efficiency and fairness to all users. An algorithm in [19] applies ACO to multicellular environment with disjoint graphs representing individual cell, while vertex is represented by sub-channel and combination of user and modulation is denoted by an edge. The resource desirability and the trail factor definition follow the metaheuristic framework formalized by Dorigo et al. in [20].

Several researchers have focused their work on the interference mitigation problem in two-tier heterogeneous network. The interference in HetNet can divided into two categories namely:

- Inter-tier interference : Interference between femtocell tier and macrocell tier.

- Intra-tier interference : Interference among multiple femtocells.

The interference reduction techniques adopt either power control or radio resource management approach. The paper 21] suggests a co-ordination procedure among base-stations through exchange of control signals between user equipment and base station. The proce- 
dure adopts both resource management and power control approaches. The total available bandwidth is divided into a number of sub-channels which is considered as resource granularity at which transmissions are co-ordinated. A base station informs user equipment about the priority of traffic it intends to transmit and the sub-channels over which it would like to transmit. An user equipment then broadcasts this information along with channel conditions to neighboring base stations. Based on the information, victim user equipments to whom it may interfere, the base station decides its transmit power and sub-channels to transmit on. This method provides equivalent performance as centralized control system albeit with lower overhead communication.

Algorithm in [22] is a graph theory based dynamic sub-channel allocation algorithm to avoid interference in femtocell networks. The cells and their mutual interferences were represented as graph elements vertices and weighted edges respectively. A sub-channel is represented as a color of vertex and, sub-channel allocation is performed using graph coloring algorithm. Using directed graph, the idea is to color the vertex with highest interference from neighbors first and to allocate the color which causes least interference to the vertex. The overhead control signalling has been kept low by threshold based triggering mechanism.

A two step channel coordination scheme suggested in [23] for LTE-A femtocell network is based on carrier aggregation. Each femtocell is assigned a carrier with least possible interference during step one. Then, in the second step, more channels are attempted to be used by a femtocell in order to increase network spectrum efficiency. To ensure fairness among femtocells, only one channel is assigned to each femtocell based on the measurement of inter-cell interference. When a base station is powered on, it measures reference signal received power (RSRP) of surrounding base stations and determine a set of base stations that are likely to have an interfering relationship with it by comparing the measured RSRP with the RSRP threshold. Then a base station would be assigned a channel that is nonoverlapping with any of the interfering base-stations; if such a channel does not exist, then a channel with minimum interference is selected. Then after calculating utility function value, 
more channels are assigned to a base station in order to achieve better spectrum efficiency. Two different utility functions have been proposed; one of them focuses on achieving highest possible spectrum efficiency while another one aims for highest system throughput while ensuring proportional fairness among multiple femtocells. This scheme outperformed when compared with the performance of fully reuse scheme.

The paper 24] presents a novel method for solving channel assignment problems in hexagonal macrocellular networks with non-homogeneous demands in a 2-band buffering system. A 2-band buffering system is a cellular network where channel interference does not extend beyond two cells. The channel assignment problem with non-homogeneous demand is first partitioned into smaller subproblems, each of which has a homogeneous demand from a subset of the nodes of the original network. The channel assignment procedure for the entire network consists of a succession of multiple assignments for all of the subproblems. The proposed polynomial time approximation algorithm, when applied for benchmark problems proved to be fast and spectrum efficient.

Fractional frequency reuse (FFR) has been used as an interference management scheme which can be categorized as resource management technique. The authors in [25] propose interference management scheme under which channels are being allocated to macrocells as per FFR technique. The femtocells select the sub-channels which are not used in the macrocell sub-area where it is located. Total available channels are divided into four subchannels. The macro users were allocated the sub-channels as per FFR where center zone and the three sectored edge regions use orthogonal channels. With the initial allocation, a femtocell senses the neighboring macrocell signals upon the startup. It compares the received signal strength indicator (RSSI) of all sub-channels and chooses to transmit on the sub-channel with minimum RSSI. The simulation results show that the specified technique enhances total/edge throughputs and reduces outage probability. The authors of [25] as well proposed femtocell interference mitigation solution based on FFR. User equipments report to their serving FAP periodically. If the report reveals high interference level, adjacent 
FAPs adopt FFR in a coordinated but decentralized way. FFR divides the femtocell in cell-center and cell-edge such that cell edge of interfering femtocells use orthogonal set of sub-channels while cell center users utilize all the sub-channels. The cell edge and cell center division is dynamic. It takes place such that user equipments suffering significant interference would be classified as cell edge users. Unlike macrocell network where position of each macro base station is fixed and due to semi-static location and number of femtocells, the static interference zones can not be deployed. As per the interference mitigation technique proposed, the serving FAP learns about the interference level of its user equipments through periodic reports. If it finds interference level significantly higher, then it shrinks the cell center by pre-defined fraction of its original radius. This process is repeated iteratively until the interference level experienced by individual user is lower than the interference threshold. The simulation results [25] indicate reduction in the inter-tier and intra-tier interference and improvement in network throughput.

The authors of [26] have proposed an interference mitigation technique which combines power control schemes in conventional macrocellular network and game theory technique in non-cooperative power control. A distributed algorithm reduces transmission powers of the strongest femto interferer. Whenever the target SINR is infeasible, a user tends to transmit with maximum transmit power. As a result, this increases interference to other users in the network. After each iterative update, macrocell base station broadcasts to FAPs whether it could achieve targeted SINR or not as well a set of femtocells whose interference is higher than threshold as the dominant interferer set. To enable users achieve their minimum SINR targets, the algorithm controls the cross tier interference at macro base station. With implementation of this algorithm, a cell-edge user can achieve its target SINR even with 100 femtocells/cell site [26].

A two-tiered HetNet consisting of small cell networks (SCN) and underlying macrocell network faces challenge of interference mitigation due to spectrum sharing between network tiers. Various techniques have been implemented in the literature in order to mitigate 
interference in the heterogeneous small cell networks.

The paper [27] suggests a spectrum sharing technique called Multi-User Vandermondesubspace Frequency Division Multiplexing (MU-VFDM). This technique allows the coexistence of a downlink Orthogonal Frequency Division Multiple Access (OFDMA) macro-cell and a cognitive multi-user small-cell system in time division duplex mode. It introduces a channel estimation protocol that does not require cooperation between the two systems, but still provides harmonious coexistence between them. By the simulation, it was proved that the proposed technique was able to achieve promising rates for the small-cells while incurring a small rate loss at the macro-cell due to interference.

The authors of [28] propose distributed coordination mechanisms for controlling the cochannel interference generated by standalone femtocells in two-tier heterogeneous network. The coordination mechanism opportunistically reuse resources without compromising ongoing transmissions on overlaid macrocells, while still guaranteeing Quality of Service in both tiers. It was proved with numerical results obtained from Monte Carlo simulations, that the coordination mechanisms substantially improve the performance of macrocell networks, while benefiting femtocells as well. Being distributed strategies, spectral efficiency was substantially increased with the deployment of proposed techniques.

Authors in [29] present three power control algorithms for interference mitigation in twotier femtocell network. In the fixed power level method, all FAPs operate at the same power level. This power level is the function of density of FAPs in that region. This scheme is applicable in the network with partitioned sub-channels. The goal of femto-QoS power control approach is to ensure femto-user QoS while minimizing femto-macro interference. The basic idea is to have each FAP transmit at the minimum power required to meet a QoS constraint for the users they serve. The third scheme macro-QoS works in favor of macro users. Each FAP transmits at a power level such that limited interference is caused to the macro-users. The simulation results show reduced FAP outage probability, however femto-user data is compromised due to lower FAP power level. Thus these approaches are 
rather suitable for control channels where data rate is not a significant performance factor as in data channels.

Authors of [30] propose resource partitioning is proposed along with suitably modified cell selection criteria in order to improve the performance of heterogeneous networks comprising small and macro cells that operate in the same spectrum. The SINR based partitioning was shown to provide improved throughput experience compared to received signal strength based partitioning while satisfying desired user fairness criteria. Moreover, a soft reuse scheme was suggested wherein the hard partitioning of the spectrum was replaced with soft reuse of the previously exclusive spectrum by means of low power transmissions. This technique was shown to improve mean throughput by $50 \%$ while maintaining edge and median throughputs compared to hard resource partitioning.

A space time coded cooperative diversity protocol proposed in 31] leverages the cellular frequency reuse concept to create space and frequency diversity among pairs of adjacent base stations of small cell network. The proposed protocol consists of two phases. During the first phase, each base station transmits symbols to its own user on its dedicated frequency band, while listening to the symbols intended to the users of the other base station on another frequency band. Cognitive cooperation is implemented in the second phase, wherein each base station transmits on the two frequency bands to the scheduled users in both base stations, by means of an appropriately chosen space time code. Simulation results show that the proposed protocol yields considerable improvement over traditional direct transmission frequency reuse approaches in terms of bit error rate and outage probability.

Authors of [3] proposes a class of distributed algorithms performing the joint optimization of radio resources in heterogeneous cellular networks composed of macro and small cells. In such networks, the unpredictability of the cell and user patterns requires self-optimized schemes. The proposed solution which is inspired from statistical physics simultaneously tries to solve the problems of channel selection, user association and power control. It has been proved by simulation results that it outperforms conventional operational methods in 
terms of throughput and energy efficiency.

A two-tier network consisting femto-cells experiences more dynamically varying environment due to plug-and-play operation of femtocell access points. This leads to more complex sub-channel allocation problem. The deterministic methods based on certain rules and parameters are too complex and time consuming to solve such a problem. The techniques based on heuristics can provide an efficient solution to such problems [5]. Various heuristics such as simulated annealing [15] and [16], neural networks [7]- [10], Genetic algorithm [12] - [14, ant colony optimization [17] - [19] have been proposed for a single tier macrocellular network. While, various deterministic approaches such as spectrum sharing/partitioning techniques in [27] and [30], resource allocation algorithms in [31] and [3] and power control algorithms [29] have been applied to interference mitigation problem in HetNet comprising of small cells and underlaid macrocelluar network. To our best knowledge, heuristics based techniques have not been considered for sub-channel allocation in such networks. In this thesis, we propose ant colony optimisation based algorithm for sub-channel allocation in two-tiered network.

In addition to this, as we represent the channel allocation as a graph coloring problem as per the papers 22] and [32] and propose 'Greedy allocation' and 'SINR based allocation' algorithms for solving this problem. In the subsequent chapter, we present a mathematical model of sub-channel allocation problem and present it as an interference graph coloring problem. We define the network environment which would be utilised for simulation purposes throughout this thesis. 


\section{Chapter 3}

\section{Model and Sensitivity Analysis}

In this chapter, we define a LTE-A network model and its parameter values such as network traffic, base-station and user locations, channel model, etc. The goal of this sensitivity study is to validate the network model and to learn the impact of network parameters on the performance of the HetNet and hence on performance of channel allocation algorithms. In addition to this, we present the details regarding graphical representation of physical resource block $(\mathrm{PRB})$ allocation problem.

\subsection{Network model}

\subsubsection{LTE-A physical layer}

Being an evolved version of LTE, LTE-Advanced is expected to be backward compatible meaning that one should be possible to deploy LTE-Advanced in the spectrum already occupied by LTE with no impact on existing LTE terminals. Consequently, for a LTE terminal LTE-A network should look alike LTE network. Such a spectrum compatibility is critical for easy and cost efficient transition to LTE-A technology. LTE can be deployed in spectrum allocations of different sizes, with transmission bandwidths ranging from $1.25 \mathrm{MHz}$, suitable for initial migration of e.g., cdma2000/1xEV-DO systems, up to around 20MHz, needed to 
provide the highest LTE data rates of $300 \mathrm{Mbps}$. In order to satisfy the requirements for high peak data rate (100Mbps downlink/50Mbps uplink), spectral efficiency and flexible channel bandwidth $(1.25-20 \mathrm{MHz})$, OFDMA technology has been selected as the basis for downlink physical layer.

In OFDMA technology, number of users communicate using multiple narrow band subcarriers spread over wider channel bandwidth. These subcarriers are orthogonal to each other which eliminates intersymbol interference (ISI). Each of these sub-carriers experiences flat fading as they have a bandwidth smaller than the mobile channel coherence bandwidth. OFDMA has several advantages in a mobile access system, such as robustness to multipath and intersymbol interference, flexible utilization of spectrum, increased spectral efficiency etc. Despite its advantages, OFDMA also suffers from certain drawbacks such as sensitivity to frequency offset (due to instability of electronics and Doppler spread resulting from mobility) and high peak to average power ratio (PAPR). PAPR occurs due to random constructive addition of sub-carriers which results in spectral spreading causing adjacent channel interference. This problem could be overcome with the use of high compression point power amplifiers and amplifier linearization. These methods are feasible on base stations but are expensive on user equipments. Hence, LTE-A uses single carrier Orthogonal Frequency Division Multiple Access (SC-OFDMA) on uplink and OFDMA on downlink [33].

The number of sub-carriers $\left(N_{S C}^{\text {total }}\right)$ available range from 128 to 2048 with the total channel bandwidth of $1.25 \mathrm{MHz}$ to $20 \mathrm{MHz}$. Approximately $10 \%$ of the subcarriers are used as guard carriers. The transmission is scheduled by physical resource block (PRB) is a set of $N_{S C}^{P R B}=12$ consecutive subcarriers. Since sub-carrier spacing is $15 \mathrm{kHz}$ each PRB is $180 \mathrm{kHz}$. For this thesis, we consider channel bandwidth of $1.25 \mathrm{MHz}$ where, as per the LTE-A specifications, total subcarriers $N_{S C}^{t o t a l}$ are 128; therefore, the available subcarriers for 
modulation are calculated as per (3.3).

$$
\begin{aligned}
N_{S C}^{\text {guard }} & =N_{S C}^{\text {total }} \times 0.1=128 \times 0.1=12.8 \approx 13 \\
N_{S C}^{\text {ava }} & =N_{S C}^{\text {total }}-N_{S C}^{\text {guard }}=128-13=115 \\
N_{P R B} & =\frac{N_{S C}^{a v a}}{N_{S C}^{P R B}}=\frac{115}{12}=9.58 \approx 10
\end{aligned}
$$

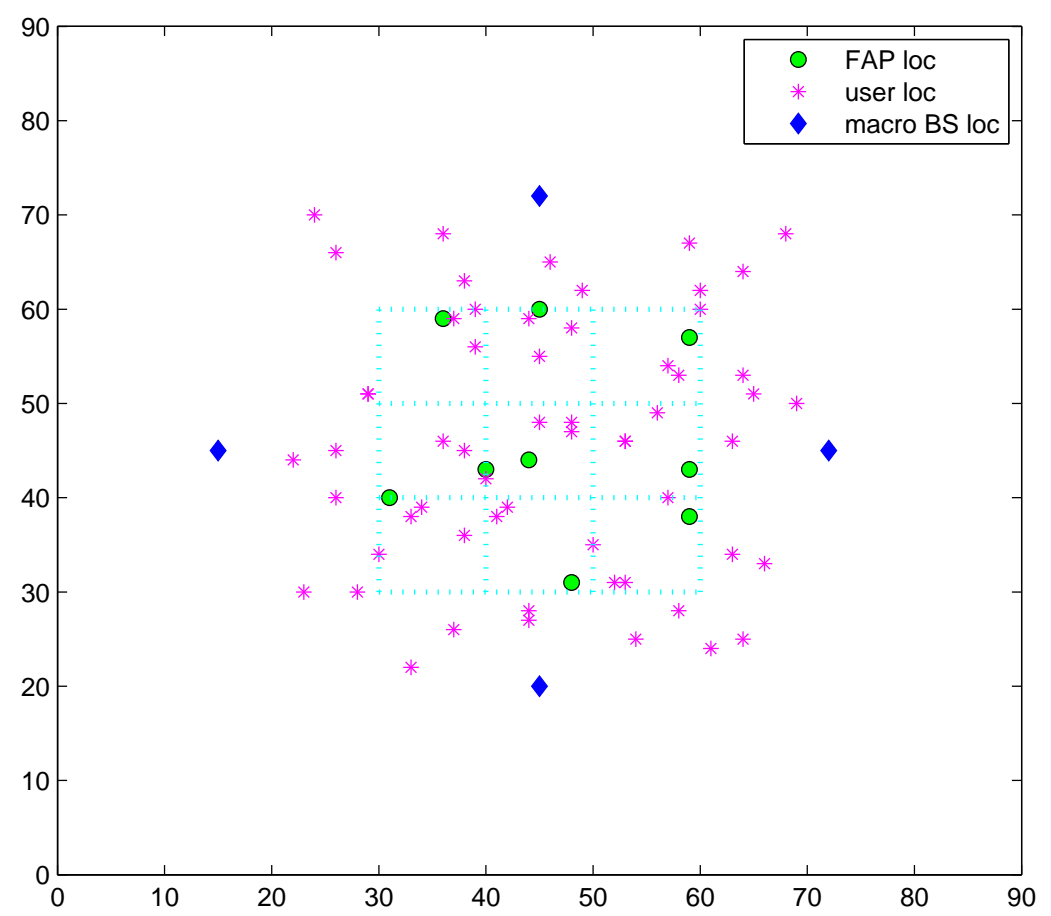

Figure 3.1: Network model with four macro and nine femto base stations

A network model for this thesis as illustrated in Figure 3.1 is a two tier small heterogeneous network. It is comprised of 9 femtocells randomly deployed in $3 \times 3$ apartment cluster where each apartment is of $10 \mathrm{~m} \times 10 \mathrm{~m}$ size and the underlaying macrocell network tier consists of 4 macrocells situated at fixed locations. Femtocells are deployed in open access mode where service access can be granted to any network user. Out of total 59 network users, 27 users are indoor users while rest of them are located outdoor. A femtocell and its three users are uniformly distributed within each apartment while outdoor users are uni- 
formly distributed around the apartment cluster. We deal with the interference mitigation problem in the downlink of LTE-A standard. As per the LTE-A specifications detailed in section 3.1.1, the downlink transmission is scheduled by PRBs where each PRB consists of 12 consecutive subcarriers. A channel of $1.25 \mathrm{MHz}$ bandwidth with $10 \mathrm{PRBs}$ are available for payload transmission. To summarize the PRB allocation problem, the 10 available PRBs needs to be allocated to 59 total network users prudently in order to improve network performance. The network model explained above has been used consistently throughout this thesis for performance analysis and comparative study of proposed algorithms.

\subsubsection{Channel model}

Four different channel models being used for system simulation are derived from the channel model used in [34]. $P_{t x}$ is the transmit power while $P_{r x}$ is the received power by a user. The path loss $P L$ is calculated by any of the equations from (3.6) to (3.9) depending on the location of user and base station type.

$$
\begin{array}{ll}
\hat{P}=P t x_{m}-P L & (\mathrm{~dB}) \quad \text { (if the basestation is macro basestation) } \\
\hat{P}=P t x_{f}-P L \quad(\mathrm{~dB}) \quad \text { (if the basestation is femto basestation) }
\end{array}
$$

The path loss between indoor users and FAP is calculated as

$$
P L_{f}^{\text {indoor }}=37+\alpha_{\text {in }} \times 10 \times \log _{10}(d)+18.3 d_{f}^{d_{f}^{2}+3 d_{f}+1.54} \quad(\mathrm{~dB})
$$

The path loss between indoor users and macrocell BS is calculated as

$$
P L_{m}^{\text {indoor }}=40+\alpha_{\text {out }} \times 10 \times \log _{10}(d)+p l_{b p} \quad(\mathrm{~dB})
$$

The path loss between outdoor users and macrocell BS is calculated as

$$
P L_{m}^{\text {outdoor }}=40+\alpha_{\text {out }} \times 10 \times \log _{10}(d) \quad(\mathrm{dB})
$$

The path loss between outdoor users and FAP is calculated as

$$
P L_{f}^{\text {outdoor }}=37+\alpha_{\text {out }} \times 10 \times \log _{10}(d)+p l_{b p} \quad(\mathrm{~dB})
$$


Here, $d$ is distance between a user and base station, $p l_{b p}$ is the building penetration loss and $d_{f}$ is indoor penetration factor between nodes determined by the number of blocking and scattering objects. The indoor and outdoor path loss exponents are respectively denoted as $\alpha_{\text {in }}$ and $\alpha_{\text {out }}$. The values of these system parameters used for simulation are described in Table 3.1

\subsubsection{System parameters}

The users and FAPs are considered to be static for the period of simulation.

\begin{tabular}{|l|l|c|}
\hline \hline Abbreviation & Parameter Description & Value. \\
\hline \hline$P t x_{m}$ & Transmit power of macrocell BS & $94 \mathrm{dBm}$ \\
\hline$P t x_{f}$ & Transmit power of femtocell BS & $55 \mathrm{dBm}$ \\
\hline$\eta$ & Noise experienced by a user (\% of total interference) & $5 \%$ \\
\hline$N_{S C}$ & Number of available subcarriers & 128 \\
\hline$N_{S C}^{P R B}$ & Number of subcarriers per PRB & 12 \\
\hline$N_{P R B}$ & Number of PRBs & 10 \\
\hline$B_{c h}$ & Channel bandwidth & $1.25 \mathrm{MHz}$ \\
\hline$N_{S C}^{\text {total }}$ & Number of total sub-carriers in the channel & 128 \\
\hline$N_{S C}^{\text {guard }}$ & Number of guard sub-carriers & 13 \\
\hline$N_{u}^{\text {in }}$ & Number of indoor users & 27 \\
\hline$N_{u}^{\text {out }}$ & Number of outdoor users & 32 \\
\hline$N_{u}^{\text {total }}$ & Number of total users & 59 \\
\hline$N_{m}$ & Number of macrocells & 4 \\
\hline$N_{F A P s}$ & Number of femtocells & 9 \\
\hline$d_{f}$ & Indoor penetration factor & 1 \\
\hline$\alpha_{\text {out }}$ & Outdoor path loss exponent & 3.5 \\
\hline$\alpha_{\text {in }}$ & Indoor path loss exponent & 2.5 \\
\hline$\gamma_{t h}$ & SINR threshold & $-5 \mathrm{~dB}$ \\
\hline
\end{tabular}

Table 3.1: System parameters.

\subsubsection{Determination of cell association}

In the heterogeneous network, the high power macro base stations can limit the amount of cell-splitting by allowing more users to associate with them. This may lead to resource constrained overloading of macrocells and under-utilization of femtocells. The benefits of cell 
association based on min path loss over the one based on max received power when applied to heterogeneous LEA-A cellular networks were demonstrated in [35]. We are following the association based on minimum path loss where a user associates with femtocell nevertheless the received power from nearest macrocell is higher. This leads to efficient cell splitting, however increased interference from nearest high power macro base station.

\subsection{Mathematical model of PRB assignment problem}

As per the metaheuristics formalised in [36], the sub-channel allocation problem which we are dealing with can be formulated as an assignment type problem (ATP). Given a number of users $\left(N_{u}^{\text {total }}\right)$ and number of PRBs $\left(N_{P R B s}^{a v a}\right)$, the problem is to determine an assignment of sub-channels to network users optimizing the objective function described. ATP can be modeled mathematically as follows:

$\max \quad f(x)=E_{\hat{x}}\left(\gamma_{i}\right)$

such that

$$
\begin{aligned}
& \sum_{j \in J_{i}} x_{i j}=1 \quad 1 \leq i \leq N_{u}^{\text {total }} \\
& x_{i j}=0 \text { or } 1, \quad 1 \leq i \leq N_{u}^{\text {total }}, j \epsilon J_{i} \\
& \hat{x}=\left(x_{11}, x_{12}, \ldots x_{21}, \ldots, x_{N_{u}^{t o t a l} N_{P R B s}^{a v a}}\right) \\
& X=\left(\hat{x}_{1}, \ldots, \hat{x}_{s}\right) \\
& S=N_{u}^{\text {total }} \times N_{P R B s}^{\text {ava }} \\
& x_{i j}= \begin{cases}1, & \text { if PRB } j \text { is assigned to user } i \\
0, & \text { otherwise }\end{cases} \\
& J_{i} \subseteq\left\{1, \ldots, N_{P R B s}^{a v a}\right\}
\end{aligned}
$$


$J_{i} \subseteq\left\{1, \ldots, N_{P R B s}^{a v a}\right\}$ is the set of admissible resources for the item $i(1 \leq i \leq n)$, whereas objective function $f(x)=E\left(\gamma_{i}\right)$ is average SINR experienced by network users. The number of additional side constraints are specific to the problem under consideration. Imposed constraints as per (3.12) and (3.13) ensure that only one PRB can be assigned to a user. A solution $\hat{x}$ which satisfies all the constraints are called feasible solution. We assume that all available PRBs can be assigned to any user therefore $J_{i}=\left\{1,2, \ldots, N_{P R B s}^{a v a}\right\} . \quad X$ is a set of possible PRB allocation solutions $(\hat{x})$ which forms the search space of combinatorial optimization problem.

\subsection{PRB allocation as graph coloring problem}

The PRB allocation problem presented in Section 3.2 can be represented as $k$-graph coloring problem where $k$ is a fixed integer and $k$ is the number of PRBs in PRB allocation problem under consideration. The graph coloring problem (GCP) can be described as follows. Given a graph $G=(V, E)$ with vertex set $V$ and edge set $E$, and given an integer $k$, a $k$-coloring of $G$ is a function

$$
c: V \rightarrow\{1,2, \ldots, k\}
$$

such that $c(i) \neq c(j)$ where $E$ contains an edge $\left[v_{i}, v_{j}\right]$ connecting vertices $v(i)$ and $v(j)$. In order to present PRB allocation problem as graph coloring problem, users will be represented as vertices and PRB will be represented as color. Here onwards in this thesis, user and vertex terms would be used interchangeably as well the terms color and PRB, e.g., an instance of network condition shown in Figure 3.1 would be transformed into a graph with all possible

edges displayed in Figure 3.2. A feasible coloring solution for such a graph with limited number of colors is not possible. Therefore, we need to set $\gamma_{t h}$, a minimum SINR performance expected at user terminals. The corresponding interference threshold $\left(I_{t h}\right)$ can be calculated as $I_{t h}=\min \left(\hat{p}_{1}, \hat{p}_{2}\right)-\gamma_{t h}[$ in $\mathrm{dB}]$ where $\hat{p}_{1}$ and $\hat{p}_{2}$ are powers received by user1 and user2 respectively. The presence of an edge is restricted to users where the mutual interference 
is higher than $I_{t h}$. The presence of an edge connecting two users signifies that the users if allocated same the PRB would cause mutual interference that is higher than $I_{t h}$. The network condition depicted in Figure 3.1 can be presented by the graph in Figure 3.2. With $\gamma_{t h}=0 \mathrm{~dB}$ constraint imposed on the graph representation, the graph would look similar to the depiction of Figure 3.3.

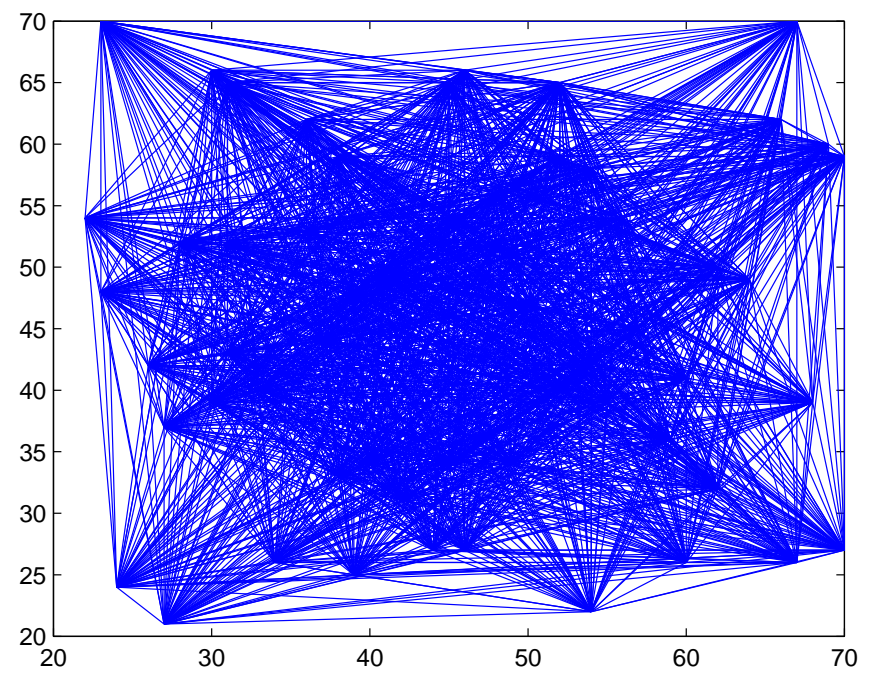

Figure 3.2: Graph with all possible edges

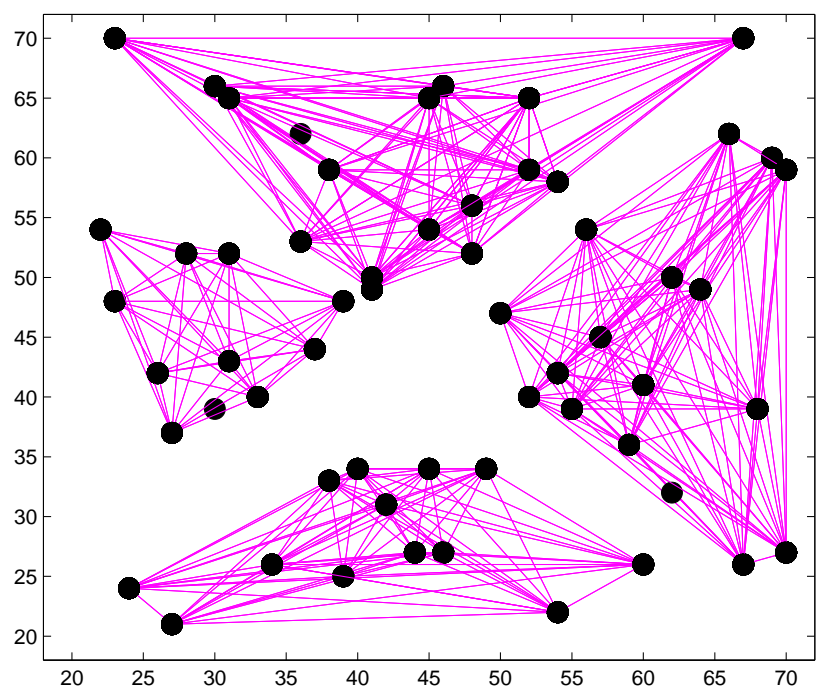

Figure 3.3: Graph with significant edges with SINR threshold $=0 \mathrm{~dB}$ 


\subsection{Sensitivity study}

The sensitivity study was performed in order to verify reliability of the network model and to learn more about the behavior of the system performance. The graphs representing PRB allocation problem, channel availability and cell association are shown in the following section.

\subsubsection{Interference graph}

The PRB allocation problem is transformed into graph coloring problem. The procedure of this conversion is described in section 3.3. For a particular instance of network condition, the quantity and position of vertices in the graph remain constant, but the number of edges varies with the value of SINR threshold $\left(\gamma_{t h}\right)$. As $\gamma_{t h}$ increases, since $I_{t h}=\min \left(\hat{p}_{1}, \hat{p}_{2}\right)-\gamma_{t h}$ [in $\mathrm{dB}]$ where $\hat{p}_{1}$ is the power received by user 1 and $\hat{p}_{2}$ is the power received by user $2, I_{t h}$ deceases. An edge is present between two vertices if mutual interference is higher than $I_{t h}$, hence the number of edges increases with increase in $\gamma_{t h}$ for $\gamma_{t h}>-5 d B$ as inferred from Figures 3.4 - 3.8. The higher number of edges means more constraints on PRB allocation, hence we can conclude that the complexity of PRB allocation problem increases with increase in the value of SINR threshold. 


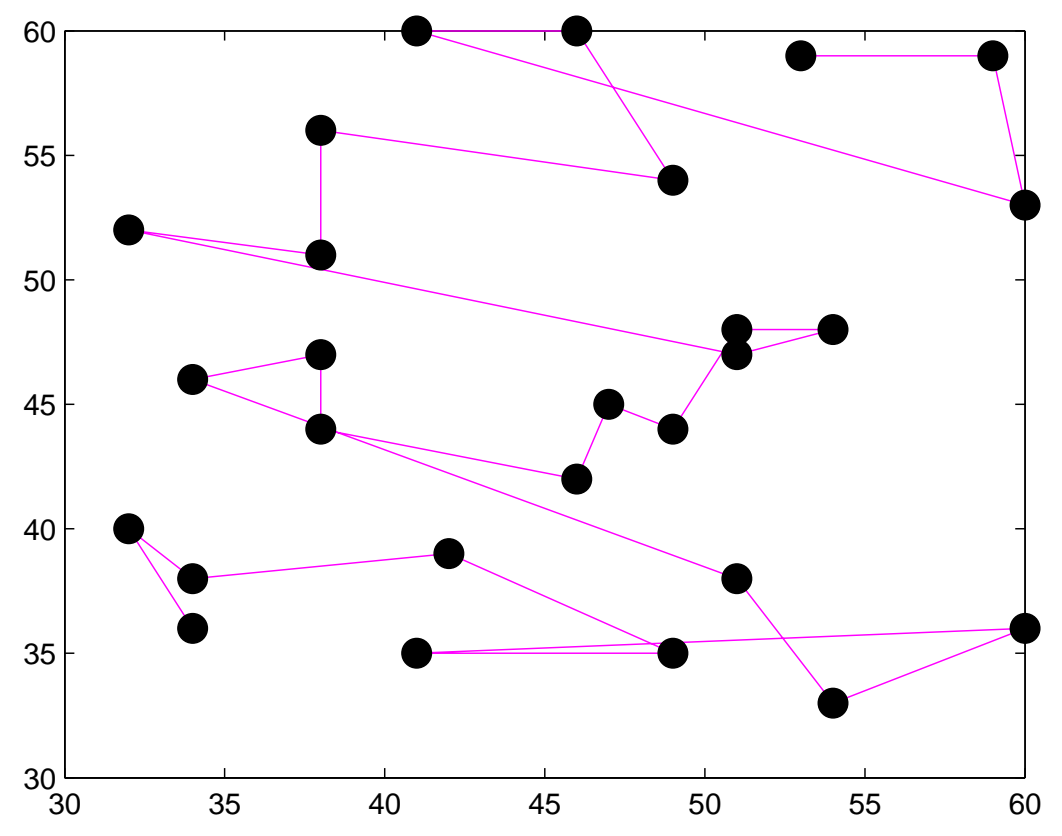

Figure 3.4: Graph with SINR threshold $=-10 \mathrm{~dB}$

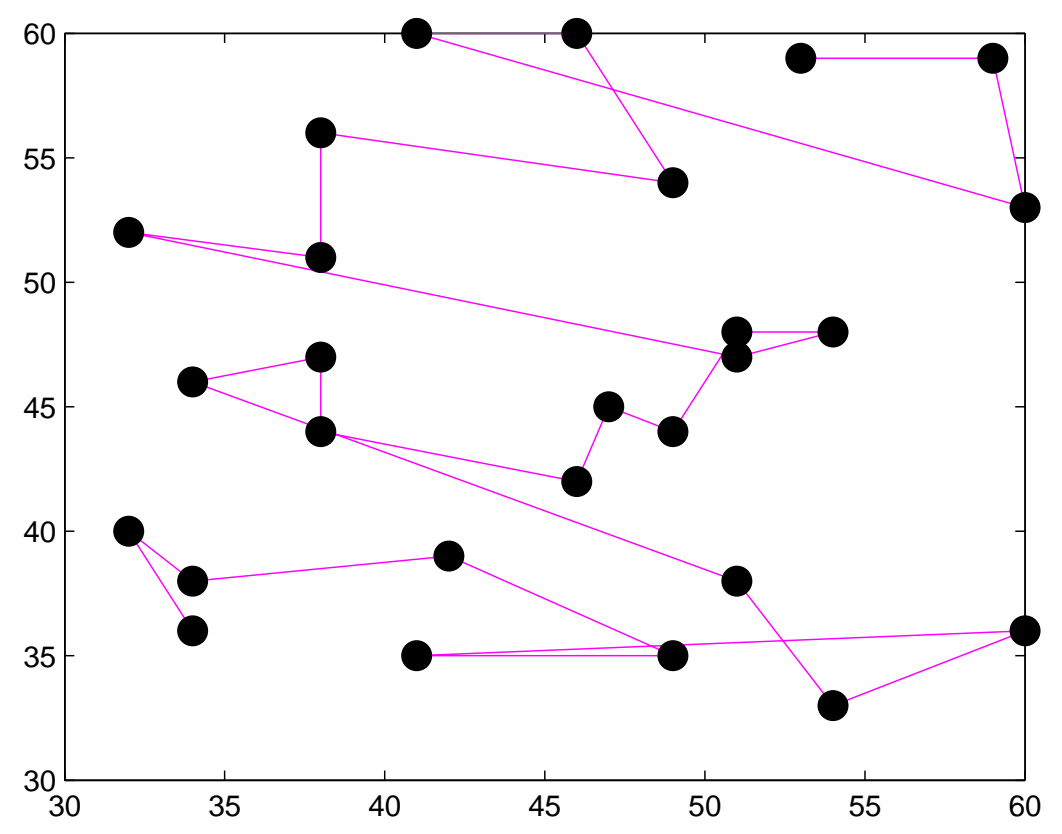

Figure 3.5: Graph with SINR threshold $=-5 \mathrm{~dB}$ 


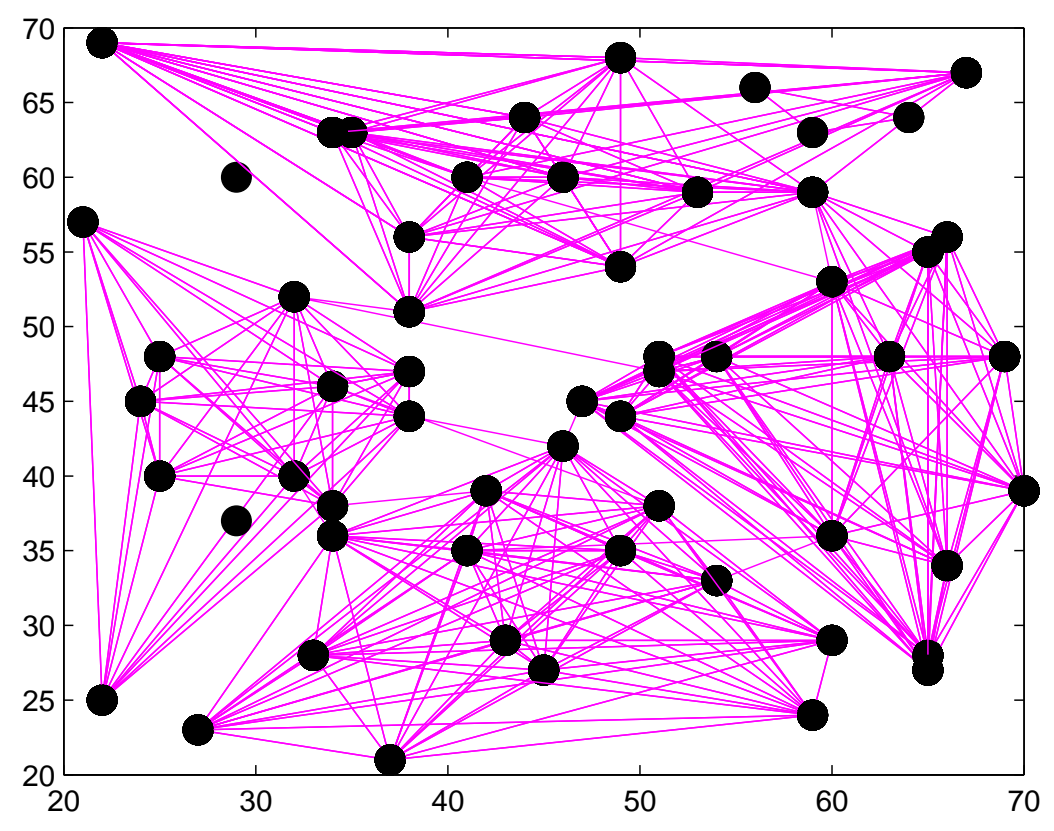

Figure 3.6: Graph with SINR threshold $=0 \mathrm{~dB}$

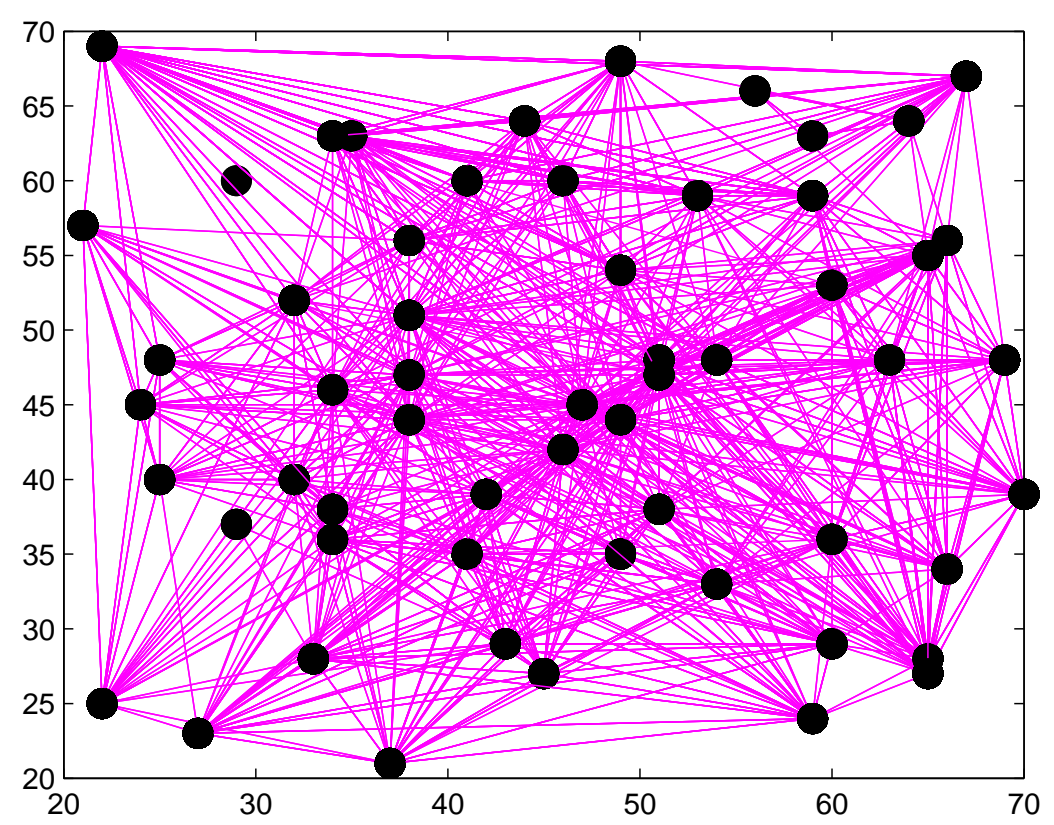

Figure 3.7: Graph with SINR threshold $=5 \mathrm{~dB}$ 


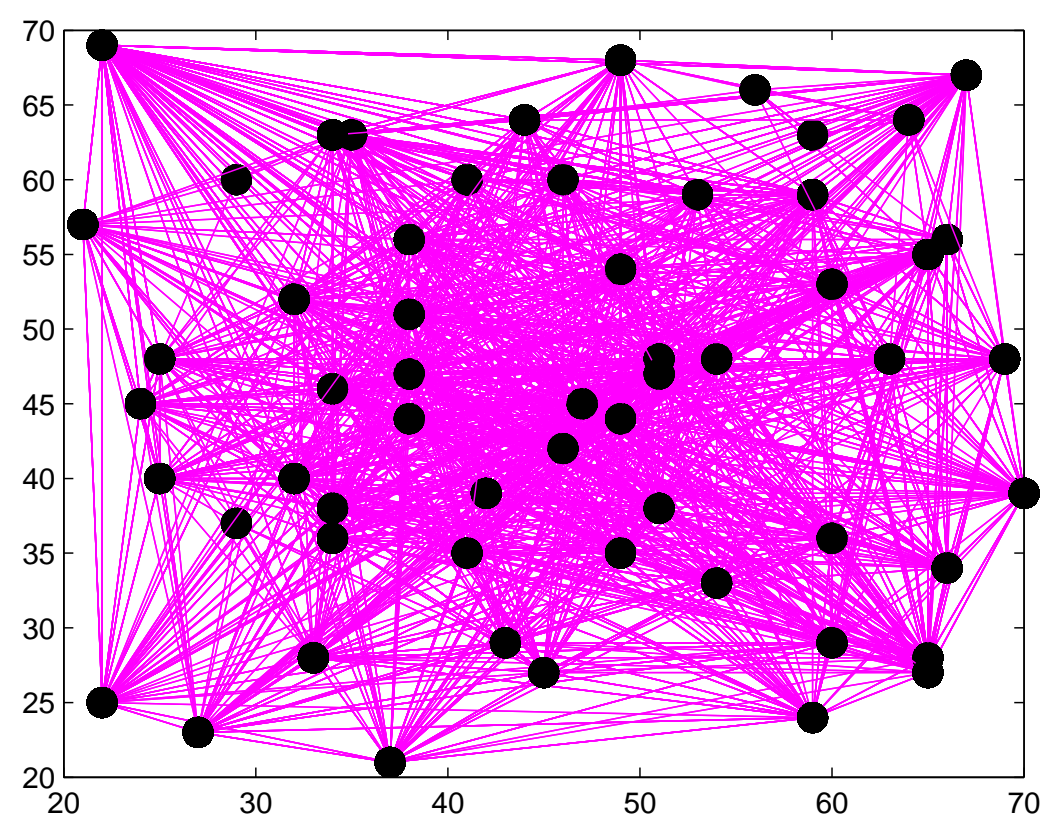

Figure 3.8: Graph with SINR threshold $=10 \mathrm{~dB}$

\subsubsection{Channel availability}

With no restrictions on the allocation of resources, the total number of communication channels available to a user in the network is $N_{P R B}$. This forms an upper bound on average number of channels that are available to a user. With the pragmatic restrictions, the numbers of channels available to a user is calculated as in (3.20).

$$
\begin{aligned}
N_{c h}^{\max } & =N_{P R B} \times 2 \\
N_{c h}^{i} & =\frac{N_{P R B} \times 2}{N_{\text {edges }}^{i}+1} \\
\psi_{i} & =\frac{N_{c h}^{i}}{N_{c h}^{\text {max }}} \times 100=\frac{100}{N_{\text {edges }}^{i}+1}
\end{aligned}
$$

In a factual network implementation, the number of channels available for a user is less than this upper bound and it is inversely proportional to the value $\gamma_{t h}$ which can be calculated as per (3.20) where $i$ is a user index. Figure 3.9 depicts this fact. The $\mathrm{X}$ axis of graph in Figure 3.9 represents SINR threshold values ranging from $0 \mathrm{~dB}$ to $20 \mathrm{~dB}$ in the steps of 
$2 \mathrm{~dB}$ and $\mathrm{Y}$ axis displays corresponding percentage of channels available to an average user $i\left(\psi_{i}\right)$ which is determined as per (3.21). For different values of outdoor path loss exponent $\left(\alpha_{\text {out }}\right)$, average percentage of channels available are calculated and represented by different line graphs of the figure. As $\gamma_{t h}$ increases, number of edges in the graph $\left(N_{\text {edges }}^{i}\right)$ which corresponds to number of users who could not be assigned the same PRB increases; thereby, average channel availability reduces. Similarly, as outdoor path loss exponent increases, a signal attenuates at higher rate and this reduces interference experienced by a user from other base stations. This leads to increase in SINR experienced by a user and hence reduction in the number of edges in the graph. Being inversely proportional to number of edges in the graph, thus the average number of channels available to a network user increases which can be evidenced in Figure 3.9.

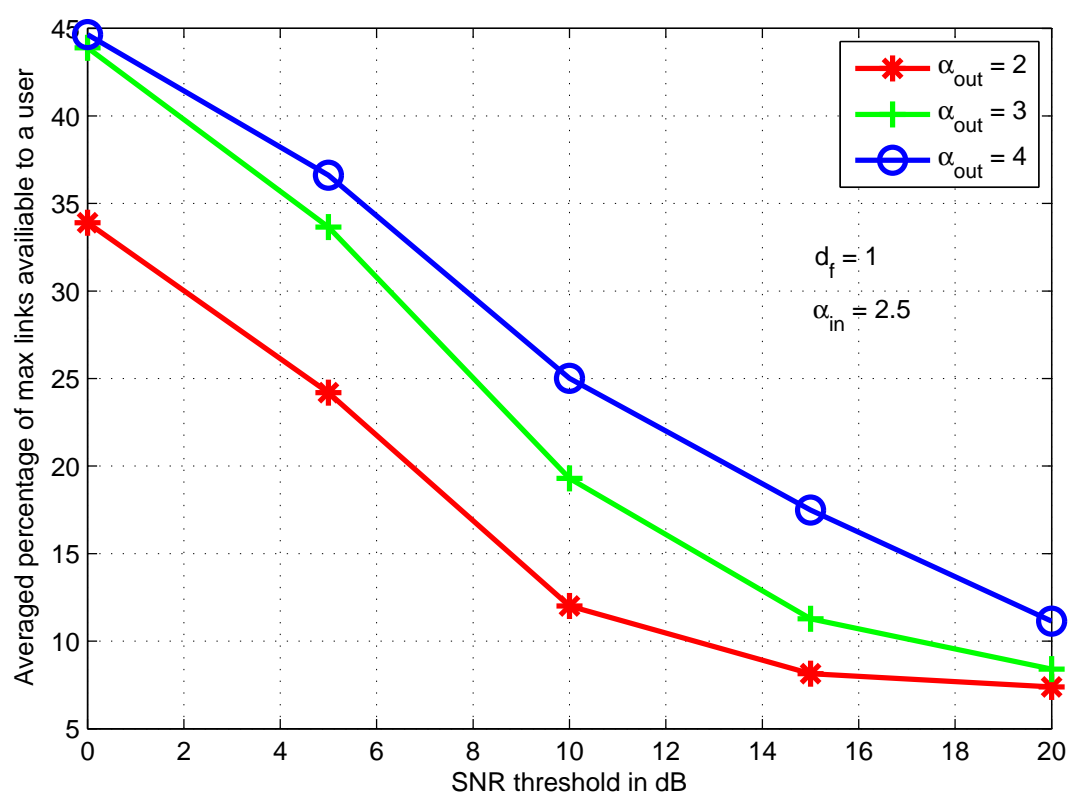

Figure 3.9: Available channels sensitivity to SINR threshold

As per the channel model used in this thesis, the penetration factor of indoor environment $\left(d_{f}\right)$ is considered while calculating path loss between indoor users and FAP. The path loss between FAP and indoor users is increased by the factor $18.3 d_{f}^{d_{f}^{2}+3 d_{f}+1.54}$. As evident from Table 3.2 , increase in $d_{f}$ by 0.1 beyond 1 increases path loss by the power of 2 . With the 


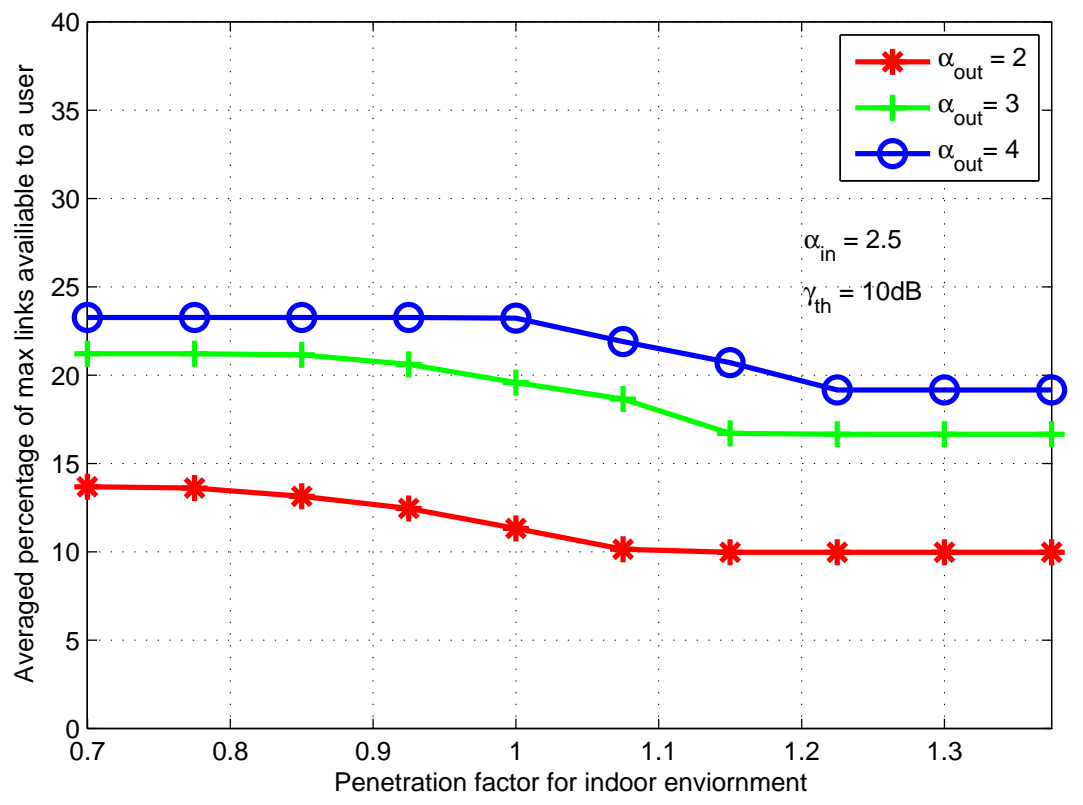

Figure 3.10: Available channels sensitivity to penetration factor of indoor environment

heavy path loss experienced by signals at FAP, majority of the indoor users get associated with macrocell as cell association criteria is based on minimum path loss. Despite the user being physically closer to the FAP, it still gets associated with macrocell (one with lowest path loss) and the FAP in closer proximity acts as a source of high interference. At this point, interference experienced by users increases, hence the number of edges in the corresponding graph. This phenomenon is depicted by reduced number of average available channels to a user in Figure 3.10 where $\mathrm{X}$ axis represents indoor penetration factor $\left(d_{f}\right)$ and corresponding number of average available channels are plotted on Y axis. The channel availability for different values of outdoor path loss exponent are depicted by three different line plots in the figure. As outdoor path loss exponent increases, path loss in the outdoor environment increases. As the radio signal attenuates at higher rate with the distance traveled, the network users on average experience reduced interference and hence the less number of edges in the graph representing network. This phenomenon is reflected in increase in channel availability. Similarly the graphs in Figure 3.11 can be interpreted. 


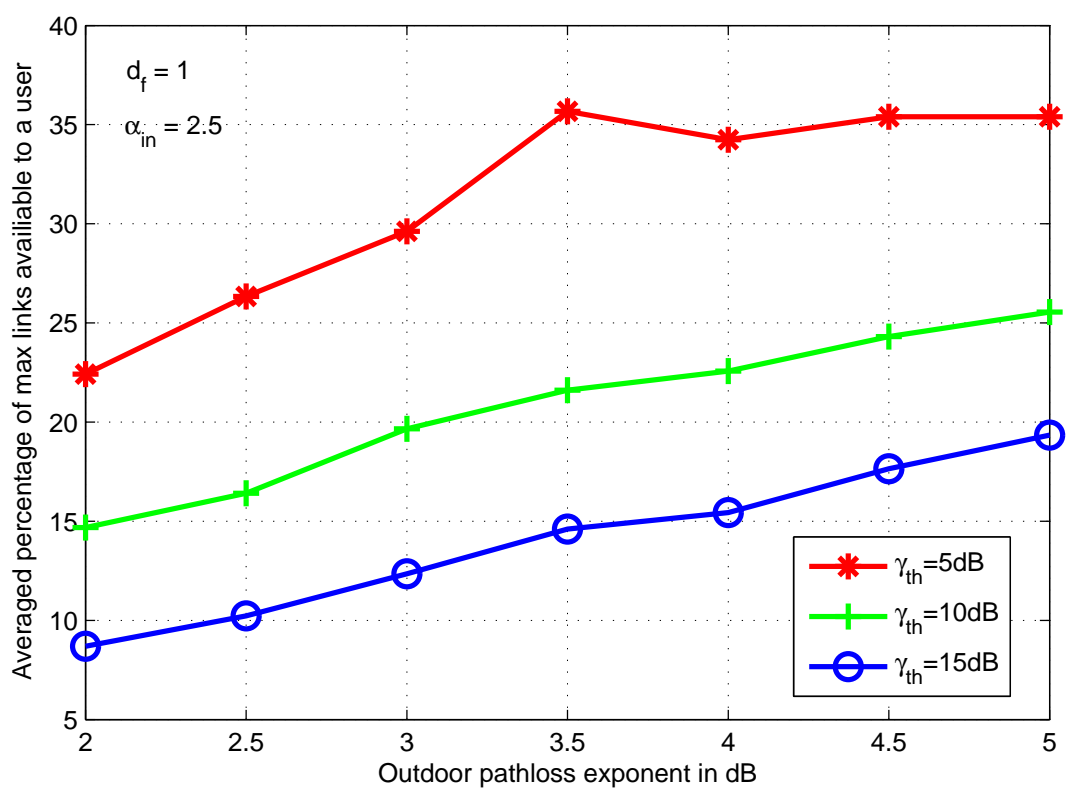

Figure 3.11: Available channels sensitivity to $\alpha_{\text {out }}$

\begin{tabular}{|c|c|}
\hline$d_{f}$ & Indoor penetration loss in $\mathrm{dB}$ \\
\hline \hline 0.7 & 4.19 \\
\hline 0.8 & 6.58 \\
\hline 0.9 & 10.74 \\
\hline 1.0 & 18.3 \\
\hline 1.1 & 32.57 \\
\hline 1.2 & 60.73 \\
\hline 1.3 & 118.81 \\
\hline
\end{tabular}

Table 3.2: Variation in indoor penetration loss with $d_{f}$

\subsubsection{Cell association}

Cell association used for network is based on min path-loss, i.e., a user is associated to the base station with minimum path loss. Therefore, it is sensitive to the network simulation parameters.

As seen from Figure 3.12, the raise in outdoor path loss exponent leads to increase in path loss of outdoor environment. Hence, more users get associated to femtocells rather than macrocells. Similarly, the increase in indoor path loss exponent leads to increase in indoor path loss. Therefore, more users get associated to macrocells as evidenced from Figure 3.13. 


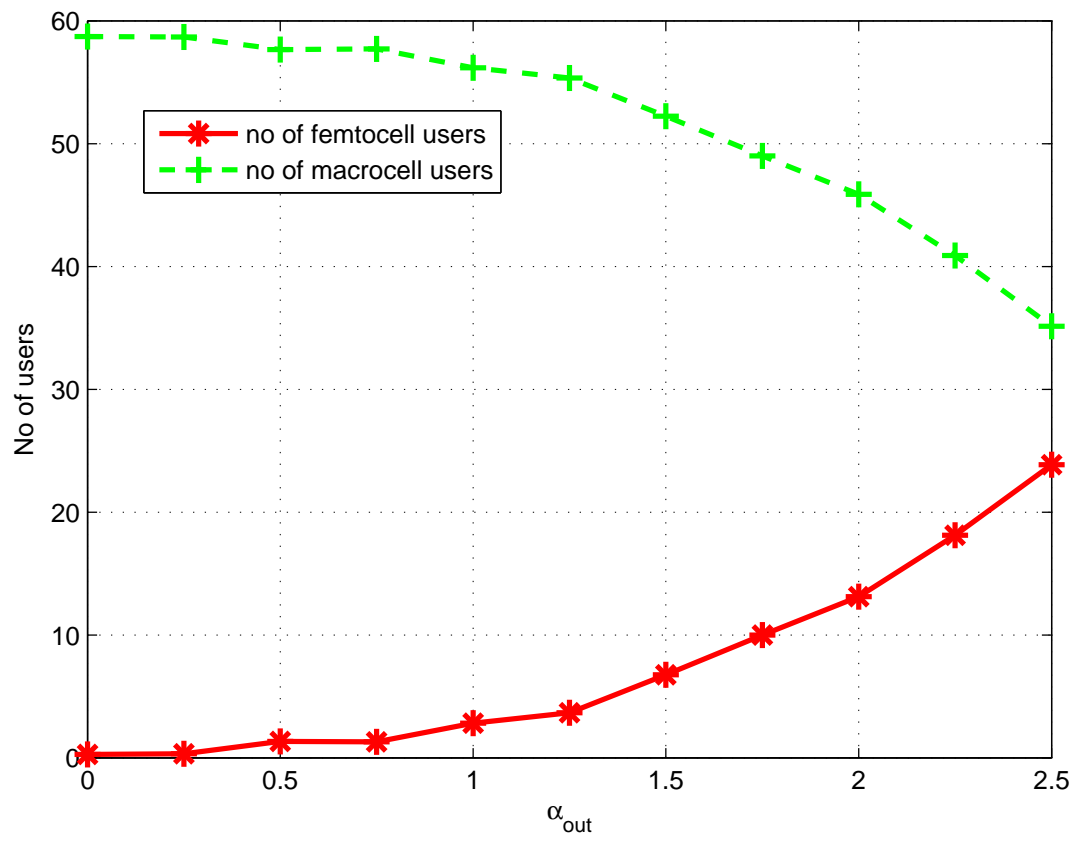

Figure 3.12: Cell association sensitivity to outdoor path loss exponent.

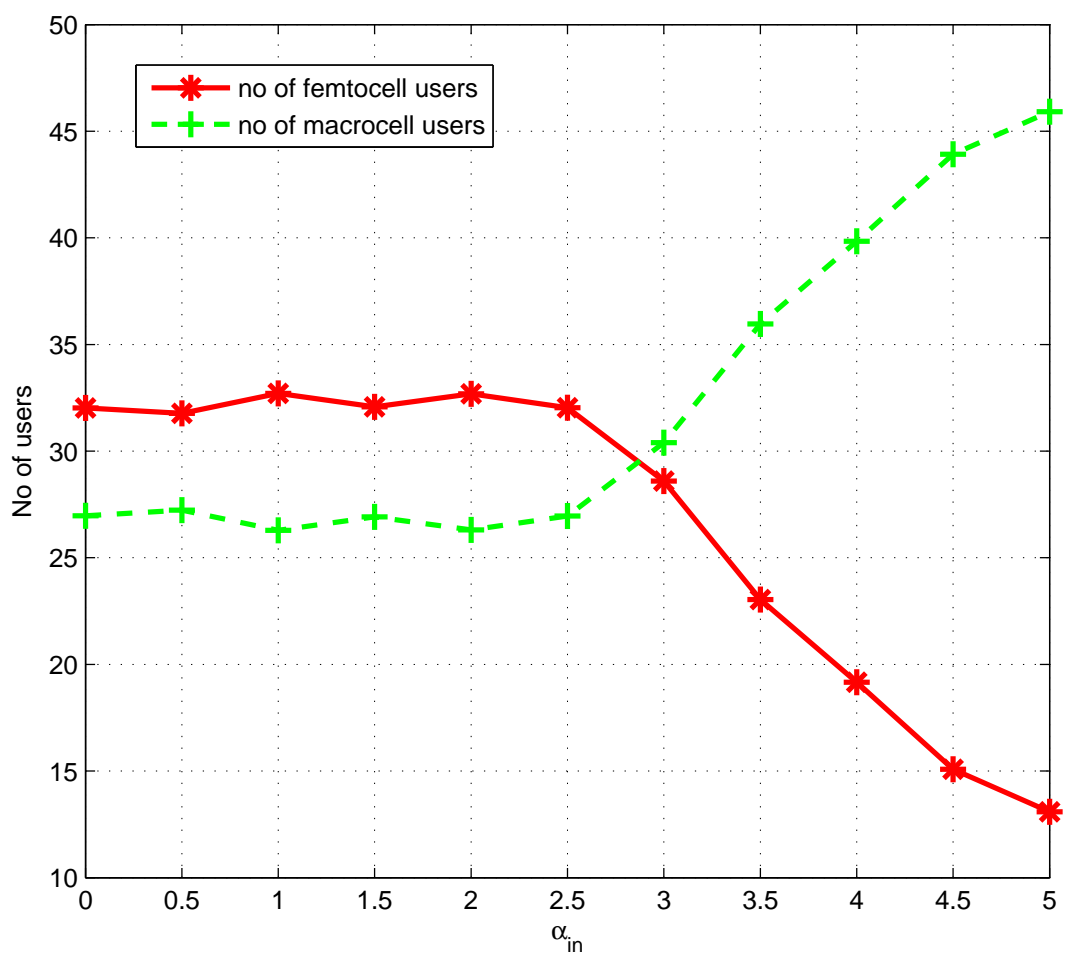

Figure 3.13: Cell association sensitivity to indoor path loss exponent. 
In this chapter, we have defined a HetNet network model which we would be utilising for simulation purposes later and have determined the suitable values of system parameters. From the analysis conducted in section 3.4.1, we can state that the interference graph representing PRB allocation problem is highly sensitive to the value of $\gamma_{t h}$. Higher the value of $\gamma_{t h}$, more complex (in terms of possible connectivity) is the PRB allocation problem. The analysis from the section 3.4 .3 affirms the suitability of selected values of parameters $\alpha_{i n}$, $\alpha_{\text {out }}$ and $d_{f}$.

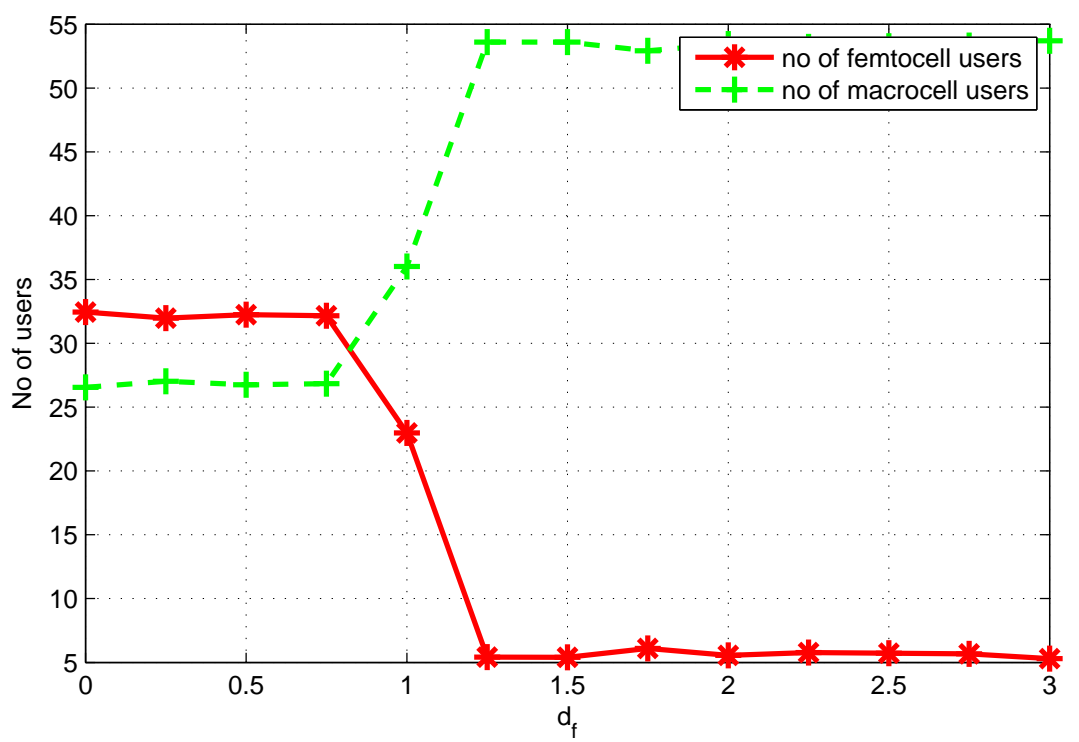

Figure 3.14: Cell association sensitivity to indoor penetration factor.

As detailed in section 3.4.2, if the value of indoor penetration factor is raised from 0 to 3 at around $d_{f}=1$ due to sudden increase in path loss, almost all of the users get associated to the macrocell network. The cell association is insensitive to any further increase in $d_{f}$ as per the graph in Figure 3.14. Hence, we have selected the value of $d_{f}$ to be 1 . 


\section{Chapter 4}

\section{Proposed Subchannel Allocation}

\section{Algorithms}

In this chapter we present the procedural details of the proposed algorithms; ant colony allocation (ACA) and greedy allocation (GA), SINR based allocation (SINRA) as well the random allocation (RA) used for comparative purposes.

\subsection{Ant colony optimization}

Ant colony optimization (ACO) is a form of swarm intelligence. Swarm intelligence (SI) is the property of a system whereby the collective behaviors of agents interacting locally with their environment cause coherent functional global patterns to emerge [37]. Inspired from foraging behavior of ant colonies, in ACO, a number of artificial ants builds solutions to considered combinatorial optimization problem and exchanges the information on the quality of solution to the ants in a next cycle of the process. 


\subsubsection{Biological inspiration}

Initially the ants move randomly in the environment, upon finding a food return to their colony while laying down pheromone trails. A pheromone is a chemical secreted by certain insects that trigger a social response in the members of same species. If an ant encounters such a pheromone trail, follows the path of trail instead of random movement. Over the time, pheromone begins to evaporate and reduces its probability that it would be followed by the ants. On the longer path, it takes a longer for an ant to travel from food source to nest and back, this leads to more evaporation of pheromone. Comparatively shorter paths encounter more trips by ants and experience denser deposit of pheromone on them making them more attractive to be chosen as path to follow by other ants. Eventually, most of the ants end up traveling on the shortest path between food source and the nest. The pictorial representation of this process is given in Figure 4.1.
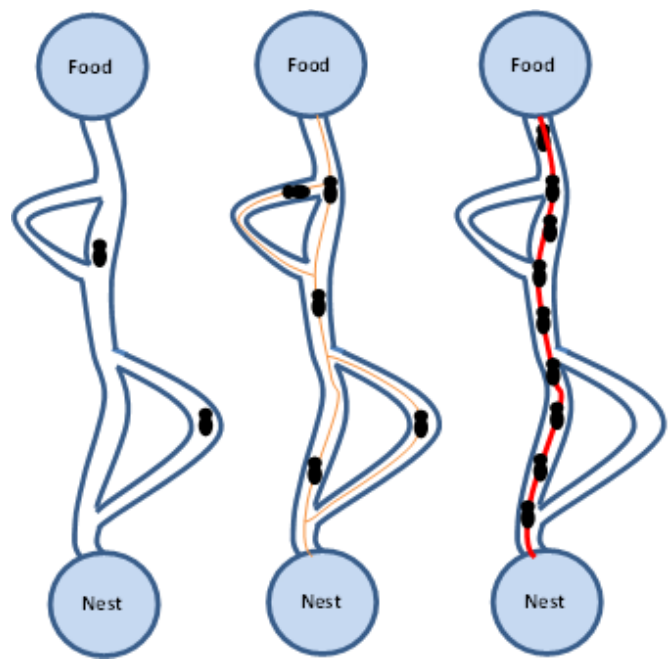

Figure 4.1: Shortest path discovery by ant colony

\subsubsection{Ant colony optimization metaheuristic}

Ant colony algorithm has been formalized into a meta heuristics for combinatorial optimization problem by Dorigo et. al [38]. In ACO, an artificial ant builds a solution by traveling across fully connected graph $G(V, E)$ where $V$ is a set of vertices while $\mathrm{E}$ is a set of edges. 
This graph is constructed from a set of solution components either by representing components vertices or by edges and an ant constructs a solution incrementally by moving from one vertex to another along the edge. The ACO meta heuristics is presented in Algorithm 1 .

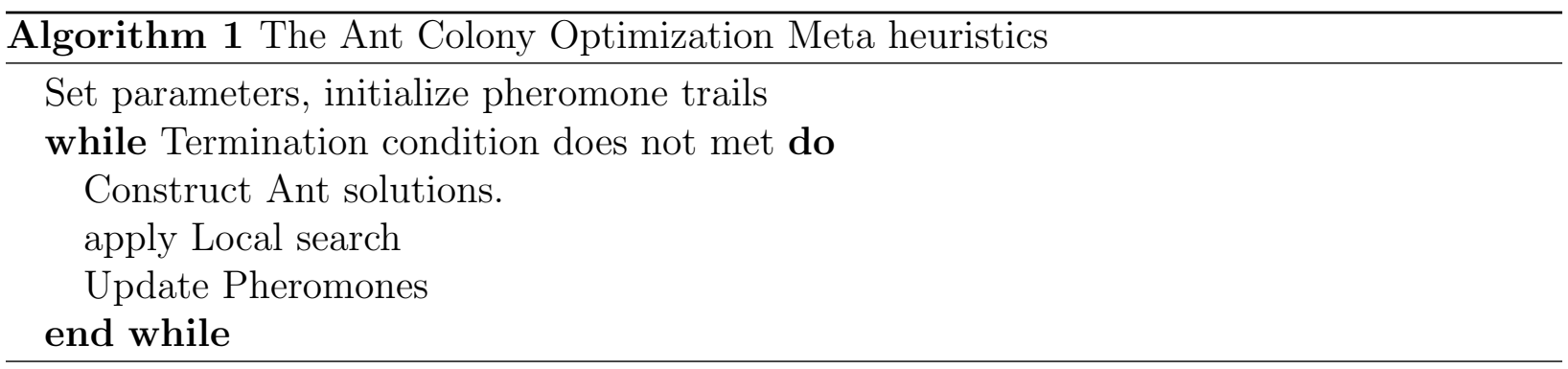

After the initial parameter setting, an algorithm iterates over three steps. During Construct Ant Solution, $N_{\text {ants }}$ number of ants construct $N_{\text {ants }}$ different solutions from the set of available solution components. Then, these solutions are improved through local search (this is an optional step) and eventually at end of the iteration pheromone is updated. In [36] authors formalize meta heuristic of ACO algorithms for the problems which can be formulated as assignment type problems (ATP). Given $n$ number of objects and $m$ number of resources, the problem is to determine the resource allocation optimizing objective function while satisfying the imposed constraints.

\subsection{ACO algorithm for PRB allocation}

The main idea behind ACO algorithm is to parallelize the search over several constructive computational threads. A dynamic memory structure incorporates the information about success of previous actions of the computational agents and guides the construction process of ants in the subsequent cycles. The main element of this algorithm is ants; unsophisticated computational agents alias ants that probabilistically and iteratively construct solution to the current problem. The intermediary partial solutions are referred as states. Each ant moves from one state to another eventually constructing a complete solution. At each step, 
every ant computes a set of feasible extensions to the current state and probabilistically chooses one of them to form a new state. For an ant, the probability of moving from state $\mathrm{p}$ to state $\mathrm{p}+1$ depends on two factors:

- A priori desirability of move from state $\mathrm{p}$ to the state $\mathrm{p}+1$ computed by the problem specific heuristic.

- A trail factor of the move indicating how proficient had this particular move been in the past to build an optimal solution.

The trails are updated at the end of each iteration, by incrementing the weight of those moves, which leads to optimal/suboptimal solution while decrementing weight of others.

The transposition of the original ant colony optimization algorithm for assignment type problem presented in [36] to our PRB allocation problem is as follows. We have $N_{P R B}$ number of PRBs available which need to be efficiently assigned to $N_{u}^{\text {total }}$. An ant colony consists of $N_{\text {ants }}$ number of ants and the algorithm runs for $N_{\text {cycles }}$ number of iterations. Each ant builds a feasible solution during each cycle. Two decisions have to be taken at each step of solution composition. First one is about the choice of a user to whom assign the resource, and the second is about the choice of resource to be assigned to the chosen user. The solutions are built probabilistically on the basis of knowledge gained from past experience of building solutions through the concept of trail factor. At each stage a of solution composition, an ant chooses an unassigned item $i$ with the probability $p r_{u s e r}(p, i)$ and assigns resource $j$ to item $i$ with the probability $\operatorname{pr}_{u s e r}^{P R B}(i, j, p)$ as per the metaheuristics detailed in 36]

$$
p r_{u s e r}(p, i)=\frac{\tau_{1}(s[p-1], i) \times \zeta_{1}(s[p-1], i)}{\sum_{q \notin\{o(1), \ldots, o(p-1)\}} \tau_{1}(s[p-1], q) \times \zeta_{1}(s[p-1], q)}
$$




$$
\operatorname{pr}_{u s e r}^{P R B}(p, i, j)=\frac{\tau_{2}(s[p-1], i, j) \times \zeta_{2}(s[p-1], i, j)}{\sum_{r \in J_{i}} \tau_{2}(s[p-1], i, r) \times \zeta_{2}(s[p-1], i, r)}
$$

In (4.1) and (4.1)

- $i$ is a user index.

- $j$ is a PRB index.

- $q$ is a user index.

- $r$ is a PRB index.

- $\operatorname{pr}_{u s e r}^{P R B}(p, i, j)$ means given a partial solution $s[p-1]$, the probability of choosing user $i$ for PRB assignment at the stage $p$ of allocation procedure.

- $\operatorname{pr}_{u s e r}^{P R B}(p, i, j)$ means given a partial solution $s[p-1]$, the probability of assigning PRB $j$ to the user $i$ at the stage $p$ of allocation procedure.

- $\zeta_{1}(s[p-1], i)$ is the desirability of choosing user $i$ for PRB allocation with a partial solution $s[p-1]$.

- $\zeta_{2}(s[p-1], i, j)$ is the desirability of assigning PRB $j$ to the user $i$ at the stage $p$ of allocation procedure.

- $\tau_{1}(s[p-1], o)$ is the trail factor of choosing user $i$ for PRB assignment at the stage $p$ of allocation procedure.

- $\tau_{2}(s[p-1], i, r)$ is the trail factor of assigning PRB $j$ to the user $i$ at the stage $p$ of allocation procedure.

- $o$ is a set of users who have be already been assigned PRB as a part of partial solution $s[p-1]$. 
- $J_{i}$ is a set of permissible PRBs for the user $i$.

At each stage $p$ of construction process, an ant calculates $p r_{u s e r}(p, i)$ as per (4.1) for all unassigned users. Given a partial solution $s(p-1)$ in which users $o(1), \ldots, o(p-1)$ have already been assigned PRBs, an ant chooses the user with maximum value of $p r_{u s e r}(p, i)$ to consider for PRB assignment. Then with chosen user $i$ an ant calculates $\operatorname{pr}_{u s e r}^{P R B}(p, i, j)$ for all PRBs available by (4.2) and assign a PRB $j$ with the maximum value of $p_{\text {user }}^{P R B}(p, i, j)$.

\subsubsection{Definition of trail factor}

SINR experienced by an average network user is used as a parameter to quantify optimality of the solution. The partial solution at the stage $p$ is quantified as the number of users that have already been assigned PRBs in $s[p-1]$ and the number of times the PRB $j$ has been already used in the partial solution. A dynamic memory structure called as trail factor matrix has been used to incorporate an information regarding how proficient had been a particular move (assignment of $\operatorname{PRB} j$ to a user $i$ ) given partial solution $s[p-1]$. A trail factor is a matrix with $N_{u}^{\text {total }}$ number of columns representing the number of times the PRB

has been already assigned in the given partial solution, while it contains $N_{u}^{\text {total }}$ number of rows representing number of users that have been already assigned PRB.

Initially all the trails (all the elements of trail factor matrix) are set to null. During each stage $p$ by each ant, in order to calculate $\operatorname{pr}_{u s e r}^{P R B}(p, i, j)$ the parameters indicating the number of times PRB $j$ has been used in the current partial solution and the number of users have already been assigned PRB are determined. The corresponding entry in trail factor matrix is used in calculation as per (4.2). At the end of each cycle, an ant with the best solution updates the trail factor matrix. For all the moves constituting the best solution, corresponding entries in trail factor matrix are updated. The updated value of a matrix element is an average of previous value and SINR experienced by implementation of the best solution from current cycle. The process sequence of an ACA algorithm is given in flowchart 4.2 . 


\begin{tabular}{|c|l|}
\hline variable & Description \\
\hline \hline $\mathrm{p}$ & solution state counter \\
\hline $\mathrm{s}[\mathrm{p}]$ & partial solution at state $\mathrm{p}$ \\
\hline $\mathrm{i}$ & user counter \\
\hline $\mathrm{j}$ & PRB counter 1 \\
\hline $\mathrm{r}$ & PRBcounter 2 \\
\hline$\tau_{1}$ & trail factor of user's selection priority for PRB allocation \\
\hline$\tau_{2}$ & trail factor of PRB allocation between a pair of user and PRB \\
\hline $\mathrm{O}=\mathrm{o}(1) \ldots \mathrm{o}(\mathrm{p}-1)$ & users with already assigned PRBs as part of partial solution \\
\hline$\zeta_{1}$ & desirability of user's selection priority for PRB allocation \\
\hline$\zeta_{2}$ & desirability of PRB allocation between a pair of user and PRB \\
\hline$J_{i}$ & a set of PRBs admissible to the $i_{\text {th }}$ user \\
\hline$p r_{u s e r}(p, i)$ & given partial solution, probability of choosing user i for allocation \\
\hline$p r_{u s e r}^{P R B}(p, i, j)$ & given partial solution, probability of allocating PRB j to user i for allocation \\
\hline BestSolutionAlgo & final solution of the ACA algorithm \\
\hline$A=V-O$ & Set of users to whom PRBs have not been assigned in current partial solution \\
\hline$N_{j}(s[p-1], i)$ & number of times the PRB $j$ has been already assigned to users adjacent to user $i$ \\
\hline$B$ & A set users adjacent to a user \\
\hline \multicolumn{2}{|l}{} \\
\hline
\end{tabular}

Table 4.1: Description of variables used 


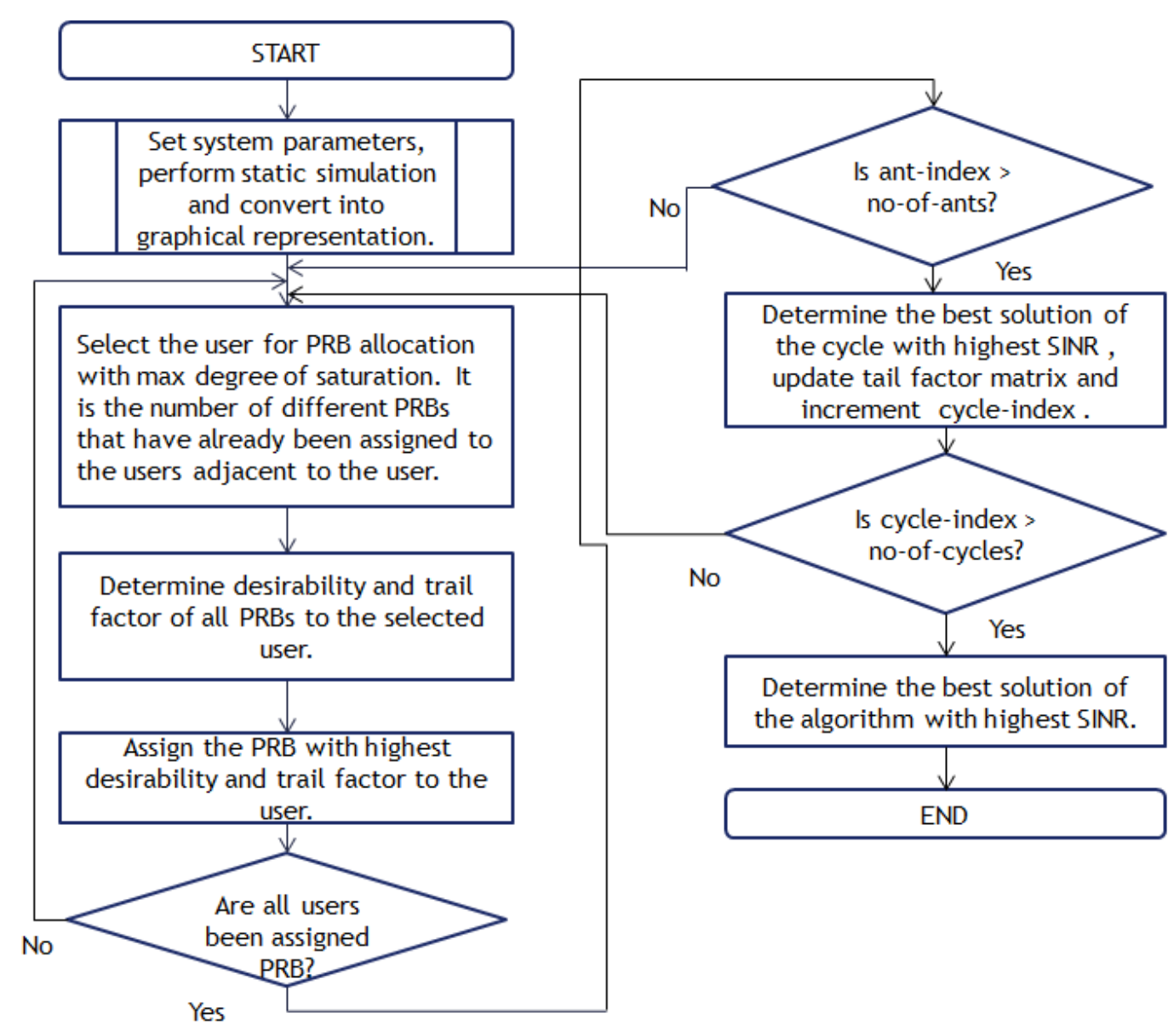

Figure 4.2: Ant colony allocation algorithm flowchart

\subsubsection{Highlights of the algorithm}

- An algorithm iterates for $N_{\text {cycles }}$ number of cycles.

- During each cycle, $N_{\text {ants }}$ number of ants generates $N_{a n t s}$ number of solutions.

- Each ant would randomly choose the first vertex which would lead to diverse solutions.

- For the assignment of first user, a PRB is selected randomly which adds more diversity to the solution space.

- The ordering of vertices can be static or dynamic. An ordering of vertices is called static if it can be decided before any assignment. The ordering of vertices is dynamic if the choice of next vertex depends on the previous assignments. After the first PRB 
assignment, vertices are dynamically selected for PRB allocation by the procedure named DSATUR proposed by Brélaz et. al. in [39]. Ties are broken randomly when the given criteria is not sufficient to order the vertices. In this procedure, $A \subset V$ where $A$ is a set of users to whom PRB has not been assigned yet. The user $i \in A$ with the maximum degree of saturation $\operatorname{deg}_{A}(i)$ has been selected. For an unassigned user $i$, the degree of saturation is the number of different PRBs that have already been assigned to the users adjacent to the user $i$. The users are said to be adjacent to each other if there exists an edge between then as explained in Section 3.4.1. This defines the desirability factor $\zeta_{1}(s[p-1], i)$ in (4.1). In our algorithm we are not taking trail factor $\left(\tau_{1}(s[p-1], i)\right)$ into consideration while ordering users to be consider for coloring priority. That means the vertices would be considered for PRB allocation in the descending order of their degree of saturation at the present state $p$ and the experience of vertices ordering from previous cycles would not be taken into consideration while ordering the vertices.

- Determination of desirability factor $\zeta_{2}(s[p-1], i)$ in calculation of $\operatorname{Pr}_{P R B}(p, i, j)$ as per $(4.2)$.

$$
\zeta_{2}(s[p-1], i)=\frac{1}{N_{j}(s[p-1], i)}
$$

where given partial solution is $s[p-1] . N_{j}(s[p-1], i)$ is number of times the PRB $j$ has been already assigned to users adjacent to user $i$.

\begin{tabular}{|l|l|c|}
\hline \hline Abbreviation & Parameter Description & Value \\
\hline \hline$N_{u}^{\text {total }}$ & Number of total network users & 59 \\
\hline$N_{\text {ants }}$ & Number of ants & 10 \\
\hline$N_{\text {cycles }}$ & Number of cycles & 15 \\
\hline$N_{P R B}$ & Number of physical resource blocks & 10 \\
\hline $\mathcal{U}$ & Uniform random variable & \\
\hline
\end{tabular}

Table 4.2: Ant colony allocation parameters 


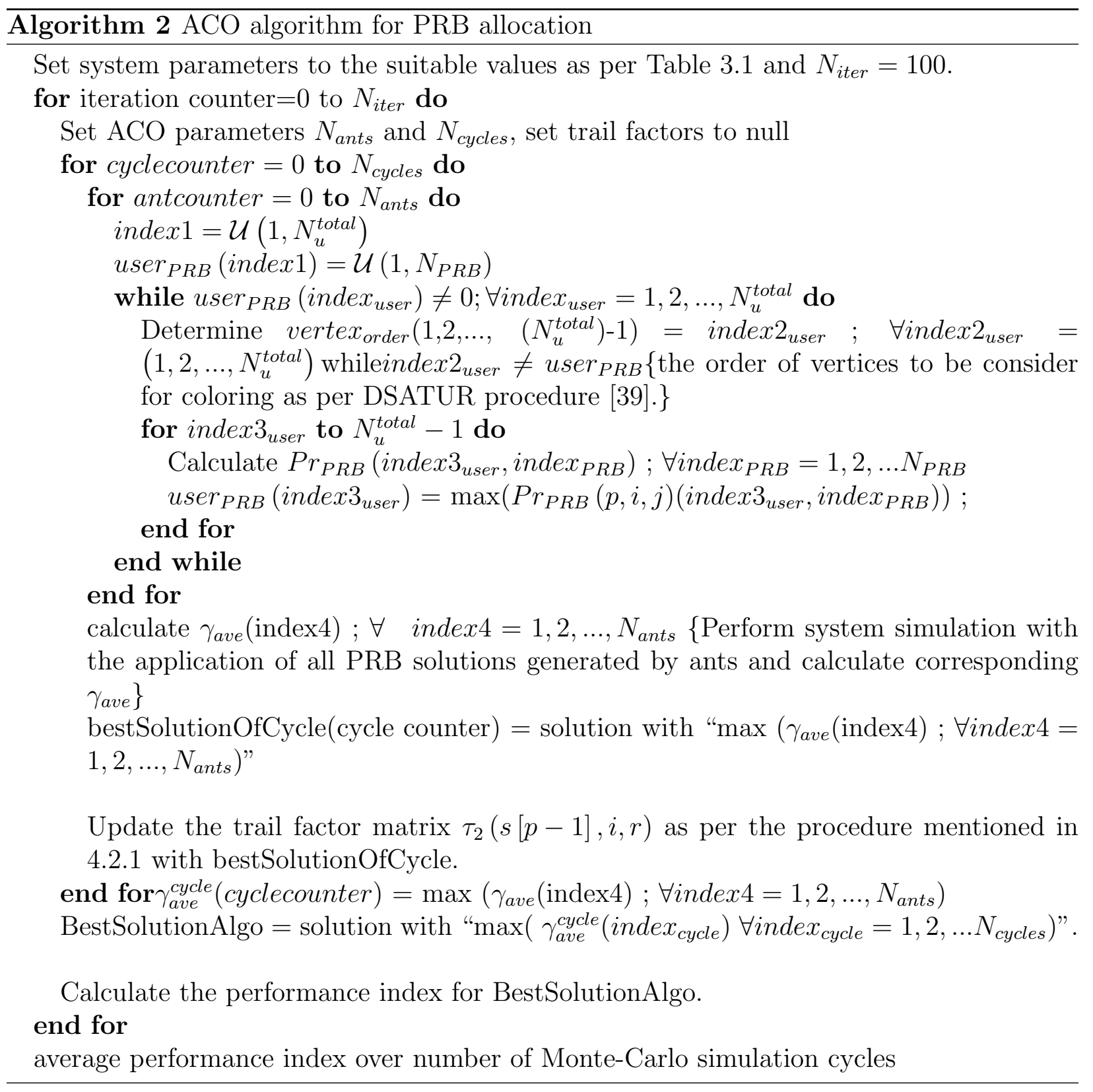


With the procedural details of the proposed algorithm in the present chapter, we will be presenting simulation results and their interpretation in the subsequent chapter with the ACA parameter values set as in Table 4.2.

\subsection{Greedy algorithm for graph coloring}

In graph theory, graph coloring is a special case of graph labeling. It is an assignment of labels called "colors" to the elements of a graph being subject to certain constraints. The graph coloring can be vertex coloring or edge coloring. A vertex coloring is a way of coloring the vertices of a graph such that no two adjacent vertices share the same color.

A greedy algorithm is an algorithm that follows a technique of making a locally optimal decision at each stage with the expectation of finding a global optimum solution for an optimization problem. It may result in optimal solution or may lead to a less than suboptimal solutions [40]. The greedy algorithm considers the vertices in a certain order $v_{1}, \ldots, v_{n}$ and for a particular vertex assigns to $v_{i}$ the smallest available color not used by $v_{i}$ 's adjacent vertices among $B \subset G ; v_{i} \notin G$ adding a new color if needed. The quality of the coloring solution depends upon the selected ordering method. The right ordering may leads to a greedy coloring with the optimal number of $\chi(G)$ colors, where $\chi(G)$ is the chromatic number of the graph $G$ which is the smallest number of colors needed to color the vertices of graph $G$ so that no two adjacent vertices share the same color.

\subsection{Greedy allocation and SINR based allocation for PRB allocation problem}

As per the details in section 3.3 , the PRB allocation problem can be represented as graph coloring problem. Here we present a greedy allocation (GA) and SINRA based (SINRA) algorithms based on the algorithmic framework of greedy algorithm for graph coloring prob- 
lem [41]. GA and SINRA algorithms follow exactly the same procedure as detailed in flowchart 4.3 and algorithm 3 except the ordering of vertices to be considered for PRB allocation.

In the greedy allocation algorithm, the vertices are considered for PRB allocation according to descending order of the power received by their respective serving base station. This means that the user with maximum received power from its serving base station would be considered first for PRB allocation. The serving base station of a network user is the base station with minimum path loss between the user and base station as described in section 3.4 .3

Along with the received power, the SINR based algorithm (SINRA) also takes interference experienced by an individual user into consideration. The ordering of users considered for PRB allocation is performed in the descending order of SINR experienced by an individual user. The SINR experienced by a network user is calculated as per (5.1) and with the assumption that all the base stations in the network transmit on the same PRB. This assumption means user experiences interference from all of the base stations in network.

The weight of an edge calculation procedure is a part of graphical representation of network condition. The weight of an edge connecting two vertices $\left(v_{i}, v_{j}\right)$ is calculated as $\max \left(I_{i}, I_{j}\right)$, where $I_{i}$ is the interference caused by the serving BS of user $v_{i}$ to user $v_{j}$, while $I_{j}$ is the interference caused by the serving base station of user $v_{j}$ to user $v_{i}$. We assume that user $v_{i}$, user $v_{j}$ and their serving BSs are the sole elements present in the network. With this assumption, the interference between two users is lowest possible interference in the network. If interference with this assumption is higher than the desired interference threshold, then interference with normal network operation would be higher than the interference threshold by all odds.

If weight of edge $\left(v_{i}, v_{j}\right)>I_{t h}$, then there exists an edge $\left[v_{i}, v_{j}\right]$ in $E$. The presence of an edge between two users signifies that, if assigned the same PRB to considered users, the interference between them would be higher than the acceptable interference threshold. 


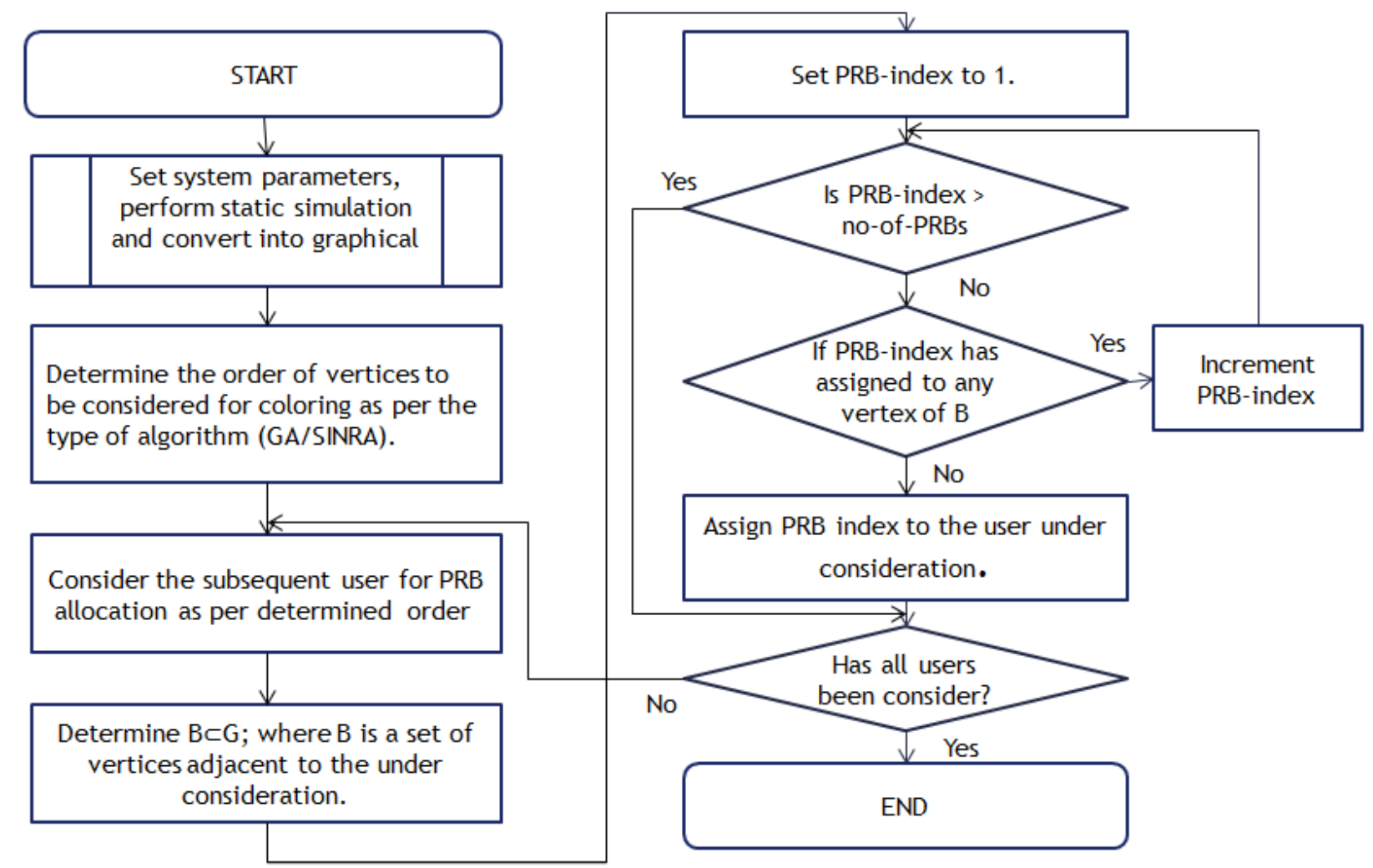

Figure 4.3: Greedy allocation/SINR based allocation algorithm flowchart

$N_{i t e r}$ is the number of iterations the algorithm has been re-executed and the performance parameter was summed up as per the Monte-Carlo method in order to nullify the effect of random variables in the system simulation.

The network condition shown in Figure 3.1 is transformed into the graphical representation as in Figure 4.4. This graph has been colored by greedy allocation algorithm as depicted in Figure 4.5. In Figure 4.5 users are represented as vertices. The presence of an edge between a pair of vertices signifies that if assigned a same PRB to them, the SINR experienced by them would be lower than $\gamma_{t h}$. The available PRBs $\left(N_{P R B}\right)=10$ are represented by 10 different colors. This figure depicts $k$-colouring of the graph where $k=N_{P R B}=10$. The vertices/users to whom algorithm could not assign a PRB due to imposed constraints have been colored in black, which represents outage probability of the allocation. 

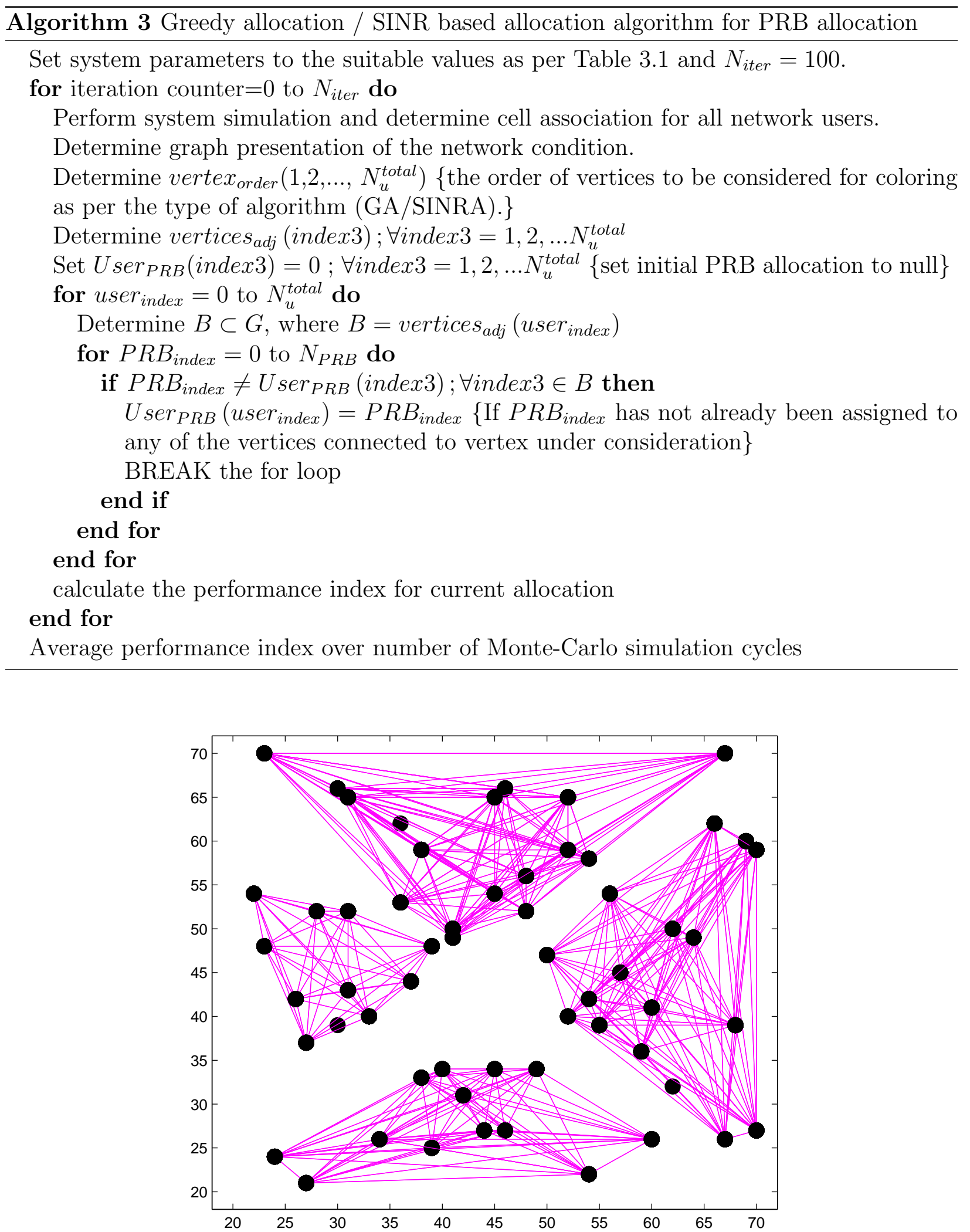

Figure 4.4: Graph with significant edges 


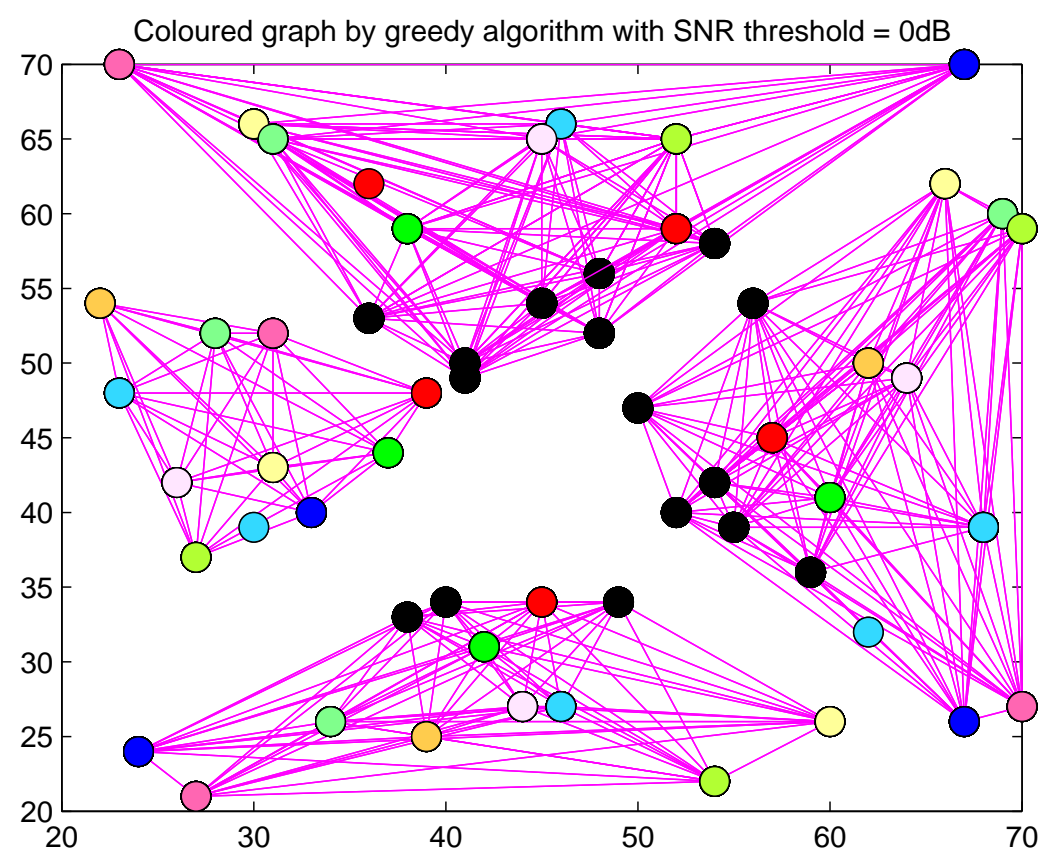

Figure 4.5: Graphical representation of solution by GA algorithm

With the prodedural details of all proposed algorithms: greedy allocation, SINR based allocation and ant colony allocation presented in this chapter, we would perform performance analysis of these algorithms in the subsequent chapter. 


\section{Chapter 5}

\section{Performance of the Proposed}

\section{Algorithms}

In this thesis, we have proposed three algorithms for interference mitigation in LTE-A heterogeneous network through PRB allocation. We have evaluated the performance of these algorithms against the parameters such as bit error rate (BER), outage probability, SINR per PRB and coverage per PRB to an average network user. To begin with, we will perform convergence analysis of ant colony allocation.

\subsection{Convergence analysis of ant colony allocation algo- rithm}

ACA algorithm is a type of evolutionary algorithm. Evolutionary algorithms are probabilistic optimization algorithms whose design is inspired by principles of biological evolution. The convergence of ACA algorithm is plotted in Figure 5.1. The average BER experienced by network users at the end of cycle is plotted against the number of cycles an algorithm has been executed for. It can be observed that an algorithm converges after 11 cycles. Thus for our simulation purpose we have executed ACA algorithm for 15 cycles in the following 
sections.

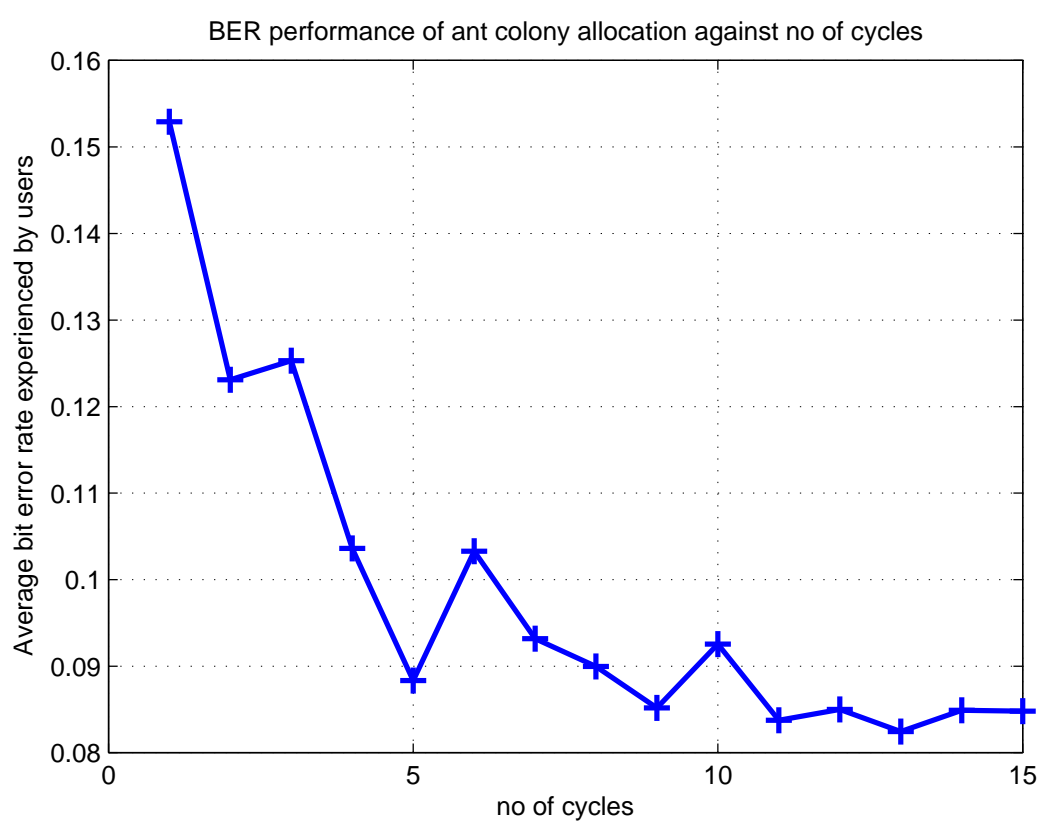

Figure 5.1: Convergence of ant colony optimization algorithm

\subsection{Comparative study of different algorithms}

In order to evaluate the performance of proposed algorithms which are greedy allocation (GA), SINR based allocation (SINRA) and ant colony allocation (ACA), they have been compared with each other. The comparative study has been conducted by quantifying the performance into various parameters such as mean SINR $\left(\gamma_{\text {avg }}\right)$, outage probability, number of PRBs utilized, SINR per PRB, coverage per PRB.

The available PRBs are allocated as per the allocation algorithm detailed in chapter 4. The allocation algorithm generates PRB allocation solution which is deployed in the simulated HetNet environment. The SINR $\left(\gamma_{i}\right)$ experienced by an individual user is determined as per (5.1). The mean value of $\operatorname{SINR}\left(\gamma_{a v g}\right)$ is calculated by averaging $\gamma_{i}$ over all network users where $\mathrm{i}=1,2, \ldots, N_{u}^{\text {total }}$. To nullify the effect of random variables in system simulation, the whole process is repeated for number of Monte-Carlo iterations and the value of 
performance parameter is averaged out. The mean values of BER calculated as per (5.2) were determined for a range of values of $\gamma_{t h}$ and plotted as in Figure 5.2.

$$
\gamma_{i}=\frac{\hat{P}_{i}}{\left(\sum_{j=1, j \neq B S_{\text {serving }}^{i}}^{N_{B S}^{t o t a l}} \hat{P}_{i}^{j}\right) \times[1+(\eta \div 100)]}
$$

where $\hat{P}_{i}=P_{t x} \times P L$

$$
\gamma_{a v g}=\frac{\sum_{i=0}^{N_{u}^{\text {total }}} \gamma_{i}}{N_{u}^{\text {total }}}
$$

where $\hat{P}_{i}$ is received power by user $i$ from its serving base-station, $B S_{\text {serving }}^{i}$ is the serving base-station of user $i$ and $\hat{P}_{i}^{j}$ is the power received by user $i$ from $j$ th base-station. $P L$ is path loss between user and base station calculated as detailed in the Section 3.1.2. $\sum_{j=1, j \neq B S_{\text {serving }}^{i}}^{N_{B S}^{\text {total }}} \hat{P}_{i}^{j}$ is the interference experienced by a user and we consider additive noise equal to $5 \%$ of the interference i.e. $\eta=5$ in (5.1).

Another performance parameter being considered is outage probability $\sigma$ which can be calculated as per (5.3) .

$$
\sigma=1-\frac{N_{u s e r s}^{\text {allocated }}}{N_{u}^{\text {total }}}
$$

where $N_{u}^{\text {total }}$ is the total number of users in the network out of which $N_{\text {users }}^{\text {allocated }}$ number of users have been allocated a PRB.

\subsubsection{Performance with change in SINR threshold}

In this section, the performance parameters were determined and plotted for various values of $\gamma_{t h}$ in the range $-10 \mathrm{~dB}$ to $5 \mathrm{~dB}$.

For a particular value of $\gamma_{t h}$ with all other system simulation parameters kept constant 


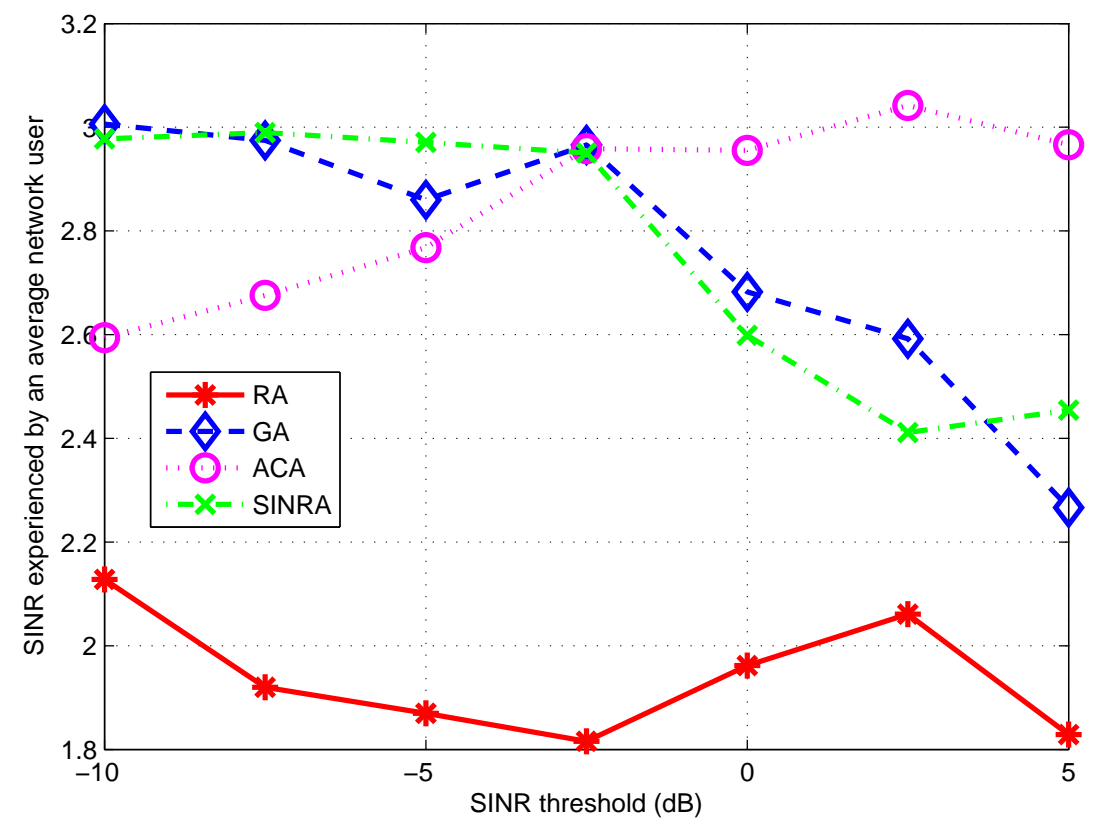

Figure 5.2: SINR vs. SINR threshold comparison of four algorithms

at suitable values, the PRB allocation is determined by the allocation algorithm. The PRB allocation was implemented on the network and SINR experienced by all network users was calculated. The mean value of SINR is calculated over all network users. As evidenced from the Figure 5.2, mean SINR thus calculated is insensitive the variation in the value of $\gamma_{t h}$ in case of RA algorithm. However with the implementation of ACA algorithm, the SNIR experienced by an average network user increases with increase in SINR threshold. The SINR experienced by an average network user decreases with increase in $\gamma_{t h}$ in the application of GA and SINRA algorithms.

As detailed in the sensitivity study of interference graphs from section 3.4 .1 around $\gamma_{t h}$ $=2.5 \mathrm{~dB}$, an increase in number of edges has been observed. Increase in number of edges means increased number of restrictions on PRB allocation; hence, more complex is the PRB allocation problem. With the implementation of ACA algorithm, SINR experienced by by a network user increases with increase in $\gamma_{t h}$. However, with GA and SINRA implementation, SINR remains constant until $\gamma_{t h}=-2.5 \mathrm{~dB}$ and decreases with further increase in $\gamma_{t h}$. From the above analysis, we can state that ACA algorithm can provide a consistent performance 
in spite of the complexity of PRB allocation problem. GA and SINRA algorithms can be classified as deterministic algorithms as they follow pre-determined rules and allocation procedure. Due to stringent constraints such as co-channel interference minimization, the channel assignment in the channel assignment becomes a difficult problem to be solved by deterministic approaches [5]. Increase in $\gamma_{t h}$ leads to increased co-channel constraints on PRB allocation problem, hence the performance of GA and SINRA algorithms degrades with increase in $\gamma_{t h}$. While ACA algorithm being an heuristic problem solving approach performs better with increased co-channel interference minimization constraints.

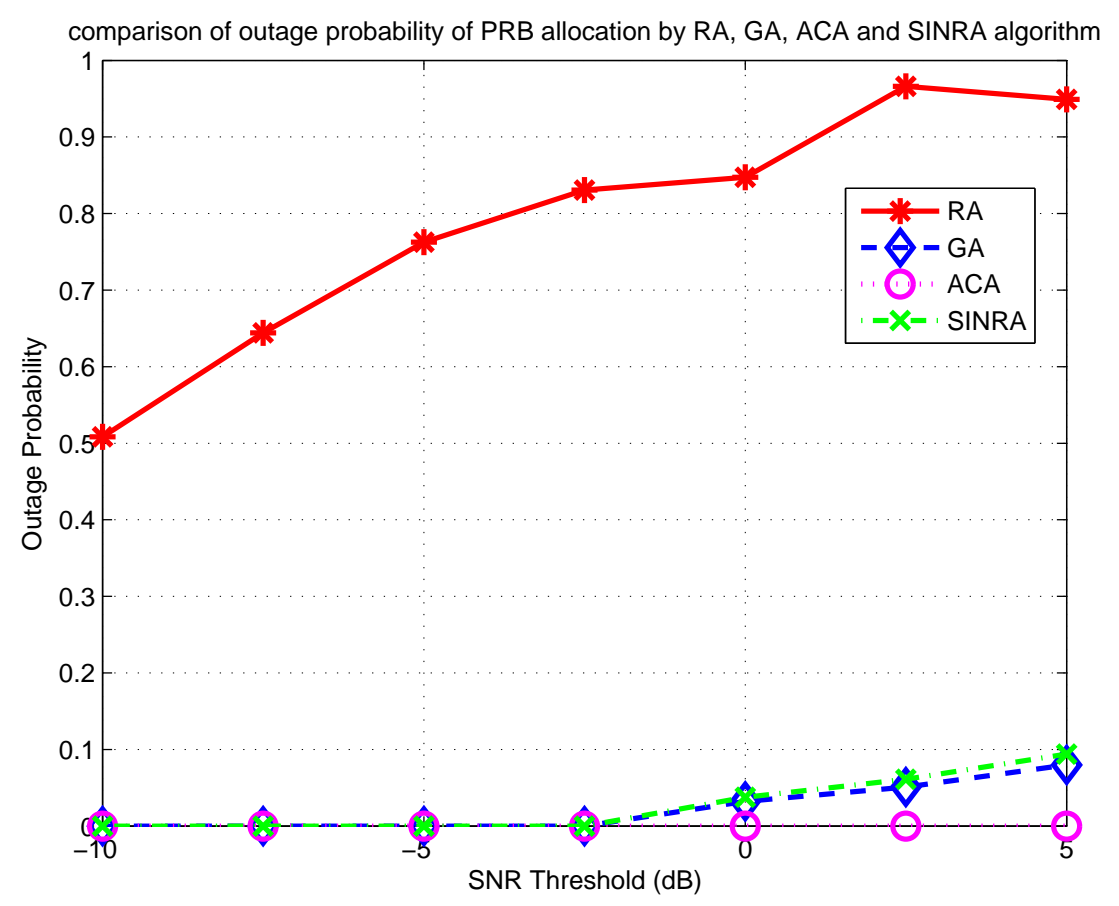

Figure 5.3: Outage probability vs. SINR threshold Comparison of four algorithms

The outage probability was calculated as per the (5.3) for the networks with each of GA, ACA, SINRA and RA algorithms deployed. For a range of values for $\gamma_{t h}$, the outage probability was calculated and plotted in Figure 5.3. With ACA deployment, outage probability is always null irrespective of the value of $\gamma_{t h}$. With GA and SINRA algorithms, for $\gamma_{t h}$ lower than $-2.5 \mathrm{~dB}$ the outage probability is almost null. Beyond $-2.5 \mathrm{~dB}$, with increase in $\gamma_{t h}$, it increases slightly reaching $10 \%$ for $\gamma_{t h}$ of $5 \mathrm{~dB}$. With the implementation of RA algorithm, 
observed outage probability is significantly high, i.e., more than $50 \%$ and increases at higher rate with increase in $\gamma_{t h}$ reaching almost total outage for $\gamma_{t h}$ value of $5 \mathrm{~dB}$.

The reason behind the varied outage probability performances of the algorithms is due to the varied allocation procedures followed by them. RA algorithm randomly assigns PRBs to the network users and calculates SINR experienced by an individual user. If any the user experiences SINR lower than SINR threshold, the previously assigned PRB would be de-assigned for the user with lowest value of SINR. This procedure is repeated until all of the network users experiences SINR higher than or equal to SINR threshold. Therefore, RA exhibits higher outage probability which is directly proportional to SINR threshold.

In GA and SINRA algorithms, a user would be assigned a PRB provided that if a PRB allocation doesn't violet the constraints of PRB allocation problem. The constraint of PRB allocation is that, no two adjacent users should be assigned a same PRB. Is none of the PRBs satisfies this constraint for a particular user then that user would not be assigned any PRB. Hence with deployment of these algorithms, outage probability takes place for the higher values of SINR threshold where more constraints are imposed on the PRB allocation problem.

The ACA algorithm, probabilistically assigns PRBs to network users depending on the desirability of a particular PRB for a user and from the past experience of similar allocation. It chooses the best solution among the number of possible solutions. Hence it covers almost all network users irrespective of the value of SINR threshold.

Figure 5.4 shows the number of PRBs utilized by GA, ACA, SINRA and RA algorithms in order to achieve the performance in Figure 5.2 and Figure 5.3. With the deployment of simple RA algorithm solution, the requirement for number of PRBs decreases with increase in $\gamma_{t h}$. Higher value of $\gamma_{t h}$ leads to increased restrictions on PRB allocation and very few users are being covered by the allocation algorithm hence reduction in the required number of PRBs. For $\gamma_{t h} \leq-2.5 \mathrm{~dB}, \mathrm{GA}$ and SINRA algorithms utilize only 3 PRBs. This means that the rest of available PRBs can be re-assigned to the network users. With the multiple 


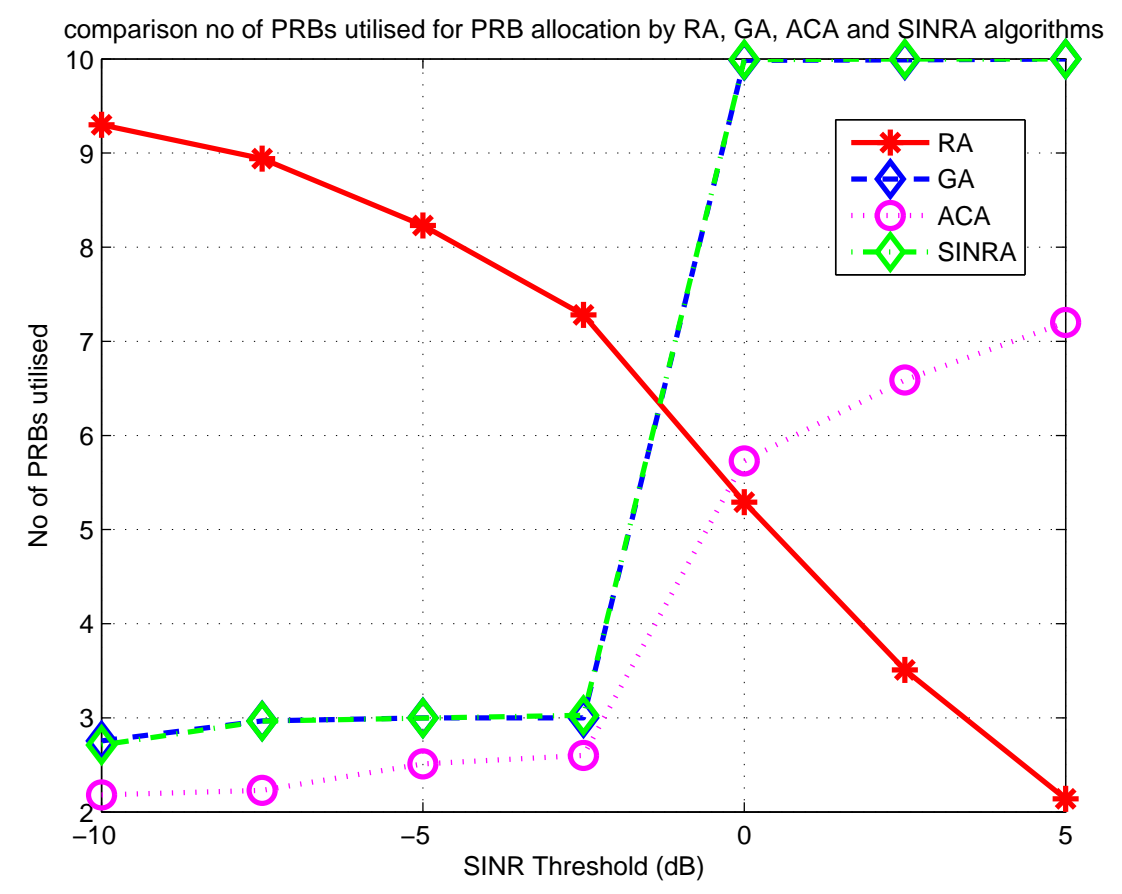

Figure 5.4: No of PRBs vs. SINR threshold Comparison of four algorithms

PRBs available for a user, it can support data communication at higher rate. ACA utilizes comparatively very few PRBS; for $\gamma_{t h} \leq-2.5 \mathrm{~dB}$ it needs only $1 \mathrm{PRB}$ and above that PRB requirement increases slowly till 7 for $\gamma_{t h}=5 \mathrm{~dB}$. For all of the proposed algorithms as $\gamma_{t h}$ increases, the number of edges in the graph representing PRB allocation problem increases thereby enhancing the restrictions on PRB allocation. This phenomenon results in more complex allocation problem consequently increasing the number of PRBs required to solve it.

To summarize we have plotted SINR per PRB and coverage per PRB performance parameters derived from the previous graphs in this section. In the graph in Figure 5.5, X axis represents $\gamma_{t h}$ values ranging from $-10 \mathrm{~dB}$ to $5 \mathrm{~dB}$ and $\mathrm{Y}$ axis displays corresponding SINR per PRB performance. As observed in Figure 5.5, RA exhibits better performance for $\gamma_{t h}>0 \mathrm{~dB}$ but it is at the expense of lower coverage. SINRA and GA algorithms exhibit equivalent SINR per PRB which decreases with increase in $\gamma_{t h}$ where complexity of PRB allocation problem increases. ACA algorithm outperforms GA and SINRA algorithms for all of the 


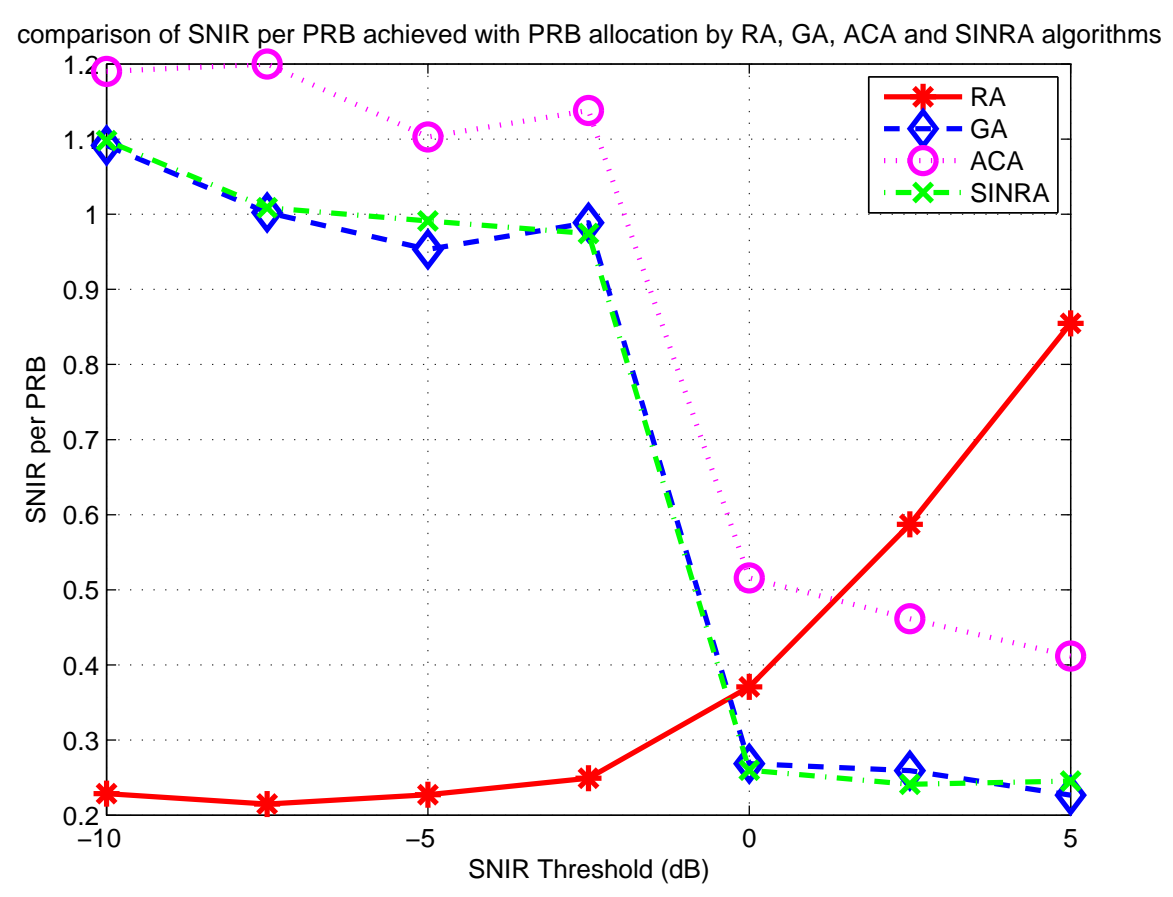

Figure 5.5: Performance parameter vs. $\gamma_{t h}$ Comparison of three algorithms

examined $\gamma_{t h}$ values.

Similar to Figure 5.5, Figure 5.6 plots coverage per PRB performance. As depicted in Figure 5.6, coverage per PRB performance reduces with increase in $\gamma_{t h}$ for the deployment of all proposed algorithms. The coverage per PRB performance of ACA algorithm is the best among all considered algorithms. The coverage per PRB performances of GA and SINRA algorithms are equivalent, though they are lower than that of ACA algorithm it is a significant improvement over RA algorithm.

From Figure 5.5 and Figure 5.6, we can conclude that the performances of ACA, SINRA and GA algorithms have been observed to be better with $\gamma_{t h} \leq-2.5 \mathrm{~dB}$. Therefore in the succeeding section the allocation algorithm performance is analyzed with variation in FAP transmit power, the $\gamma_{t h}$ has chosen to be constant at $-5 \mathrm{~dB}$ in order to achieve better performance. 


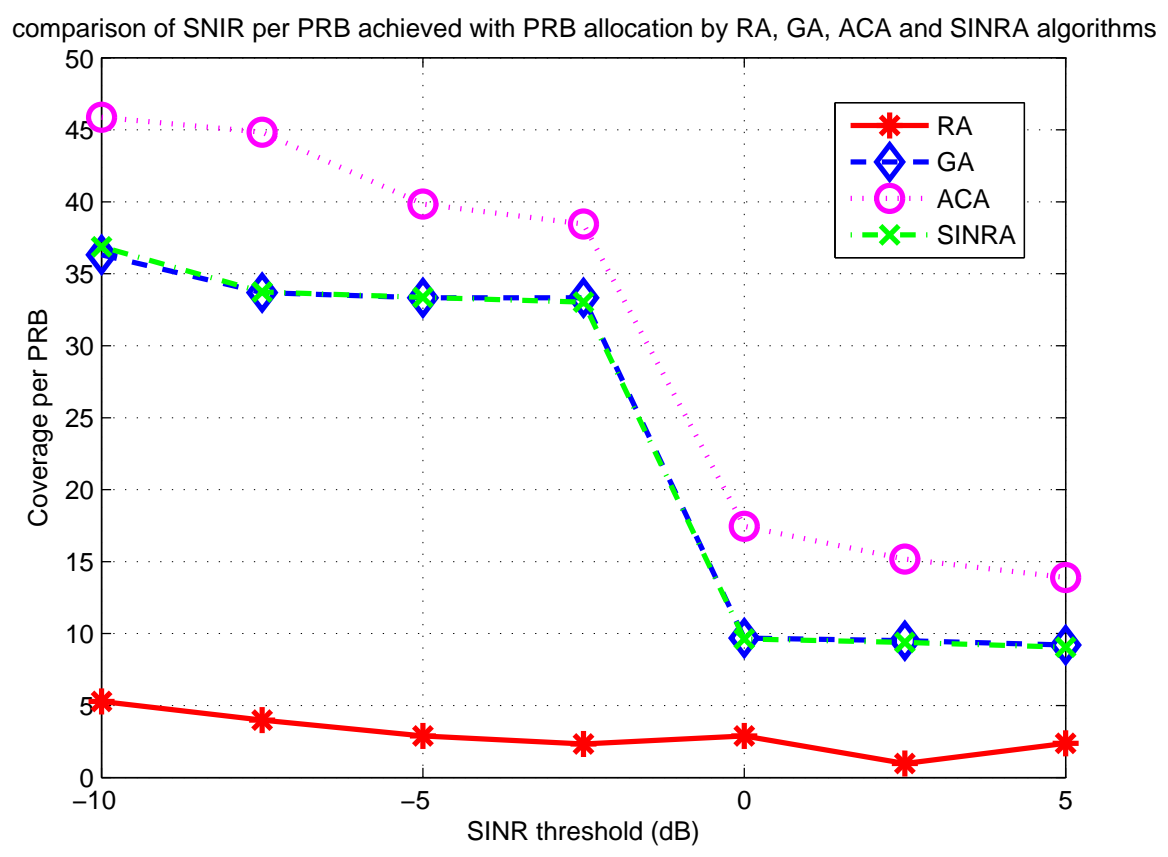

Figure 5.6: Performance parameter vs. $\gamma_{t h}$ Comparison of three algorithms

\subsubsection{Performance with change transmit power of FAPs}

For a particular value of FAP transmit power $\left(P_{f}\right)$ with all other system simulation parameters held constant at suitable values which includes $\gamma_{t h}$ of $-5 \mathrm{~dB}$, the PRB allocation is determined by the allocation algorithm. The PRB allocation was implemented on the network and SINR experienced by an individual network user $\left(\gamma_{i}\right)$ was calculated. The average SINR experienced by all network users $\left(\gamma_{\text {avg }}\right)$ is calculated over all network users as per (ㄷ.5.2).

The $\mathrm{X}$ axis of the graph in Figure 5.7 displays FAP transmit power at which all FAPs in the network transmit while the $\mathrm{Y}$ axis displays corresponding SINR experienced by an average network user $\left(\gamma_{\text {avg }}\right)$. The SNIR experienced by network users increases linearly with increase in $P_{f}$. The four line graphs represent the network performance with the implementation of each of the RA, GA, SINRA and ACA algorithms. As seen from Figure 5.7 SINR performances of all proposed algorithms are similar which are better than that of RA algorithm.

Figure 5.8 depicts outage probability experienced the network users with the change in 


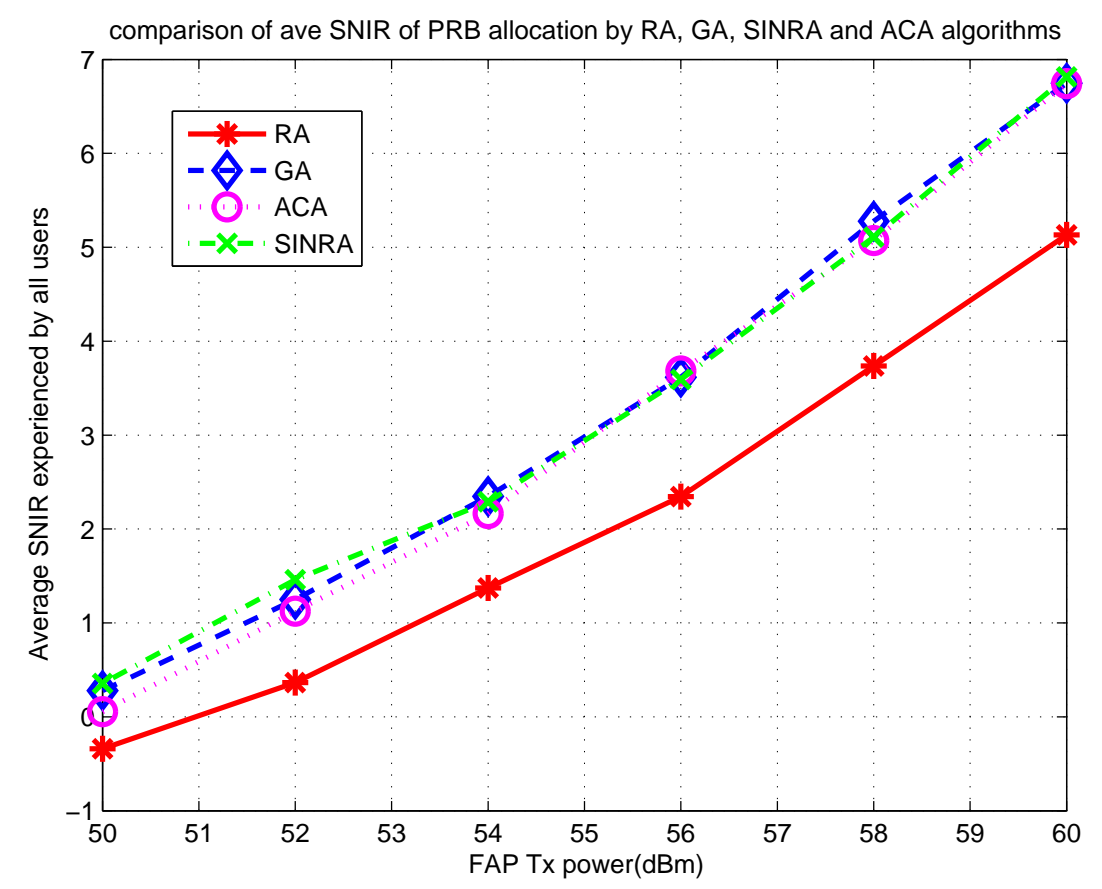

Figure 5.7: SINR vs. FAP Tx power Comparison of four algorithms

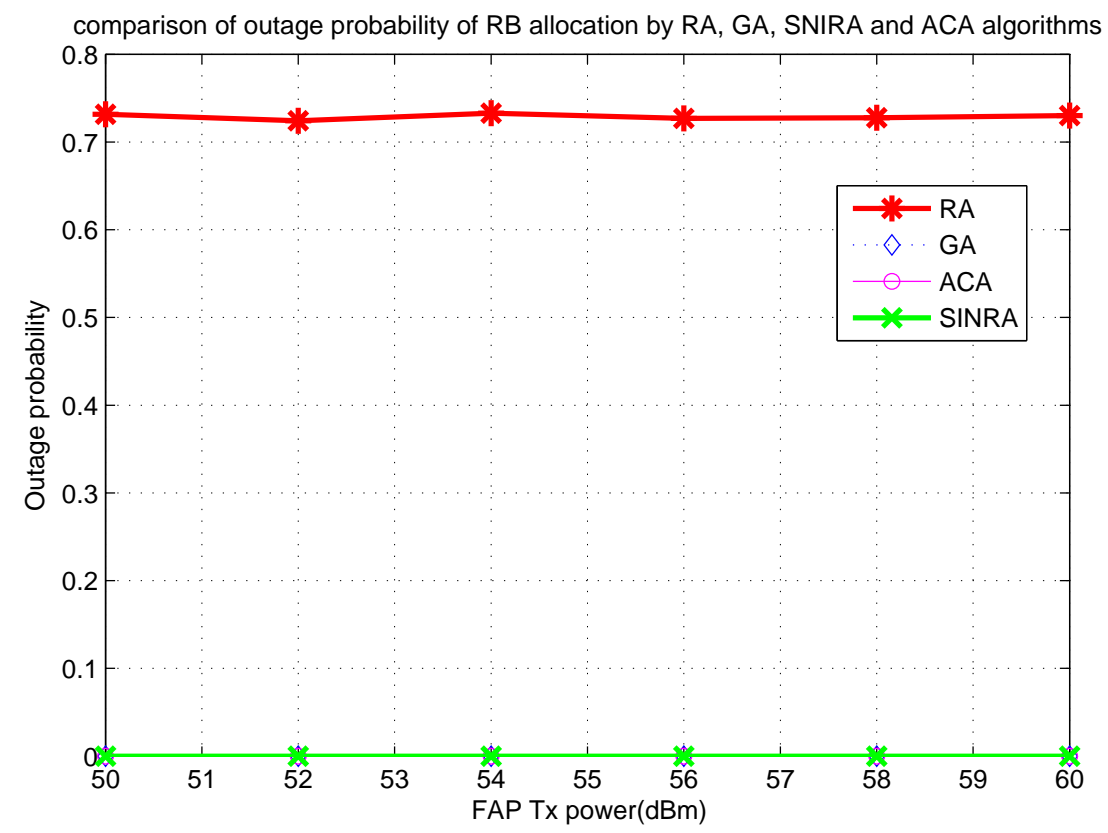

Figure 5.8: Outage probability vs. FAP Tx power Comparison of four algorithms 
FAP transmit power. All of the proposed PRB allocation algorithms serve network users with null outage probability. However, with implementation of the RA algorithm network users experience almost $70 \%$ of outage probability.

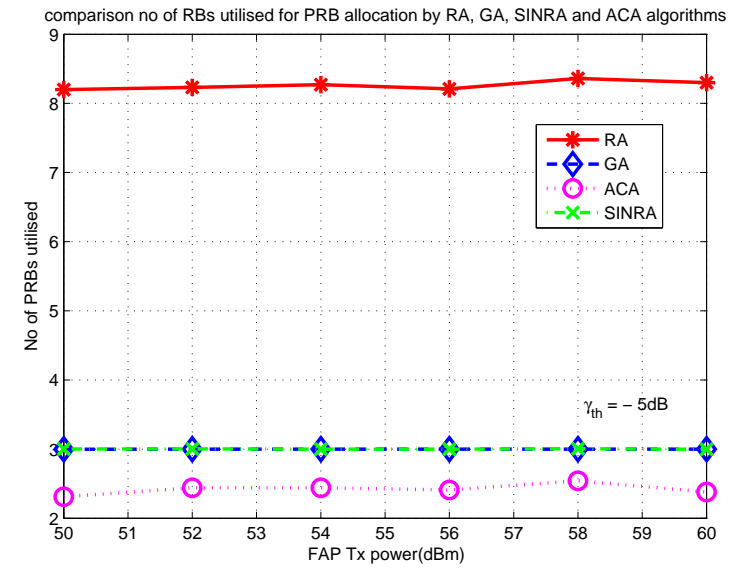

Figure 5.9: No of PRBs vs. FAP Tx power Comparison of four algorithms with $\gamma_{t h}=$ $-5 \mathrm{~dB}$

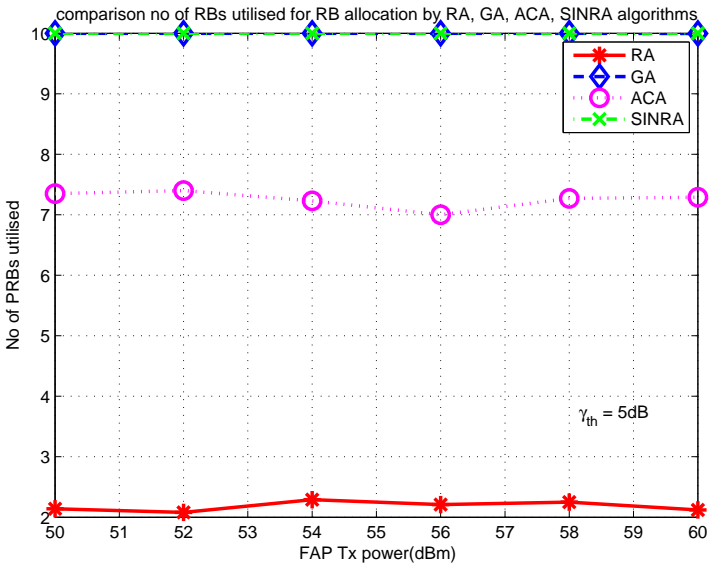

Figure 5.10: No of PRBs vs. FAP Tx power Comparison of four algorithms with $\gamma_{t h}=$ $5 \mathrm{~dB}$

In Figure 5.9, the number of PRBs utilised in PRB allocation solution are plotted against the values FAP transmit power ranging from $50 \mathrm{dBm}$ to $60 \mathrm{dBm}$ with $\gamma_{t h}=-5 \mathrm{~dB}$, four different line plots represent the responses by the four different PRB allocation algorithms under consideration. The number of PRBs utilised by GA and SINRA algorithms are $50 \%$ less than the PRBs utilised by RA algorithm. While ACA algorithm utilises almost $57 \%$ less PRBs compared to RA algorithm. Figure 5.10 depicts number of PRBs utilised with $\gamma_{t h}$ $=5 \mathrm{~dB}$ by ACA, GA, SINRA and RA algorithms. With the increase in $\gamma_{t h}$, the number of PRBs utilised by all proposed algorithm increases as the restrictions on allocation problem increases more number of PRBs are needed to solve it. With RA implementation the number of required PRBs reduces with increase in the value of $\gamma_{t h}$ from $-5 \mathrm{~dB}$ to $5 \mathrm{~dB}$. With the value of $\gamma_{t h}=5 \mathrm{~dB}$, RA provides very low user coverage hence number of required PRBs reduces.

From Figure 5.7, we can state that, as FAP transmit power increases SINR experienced by users increases. As depicted from Figure 5.8 and Figure 5.9, the number of required PRBs and percentage of users served are insensitive to variation of FAP transmit power. 


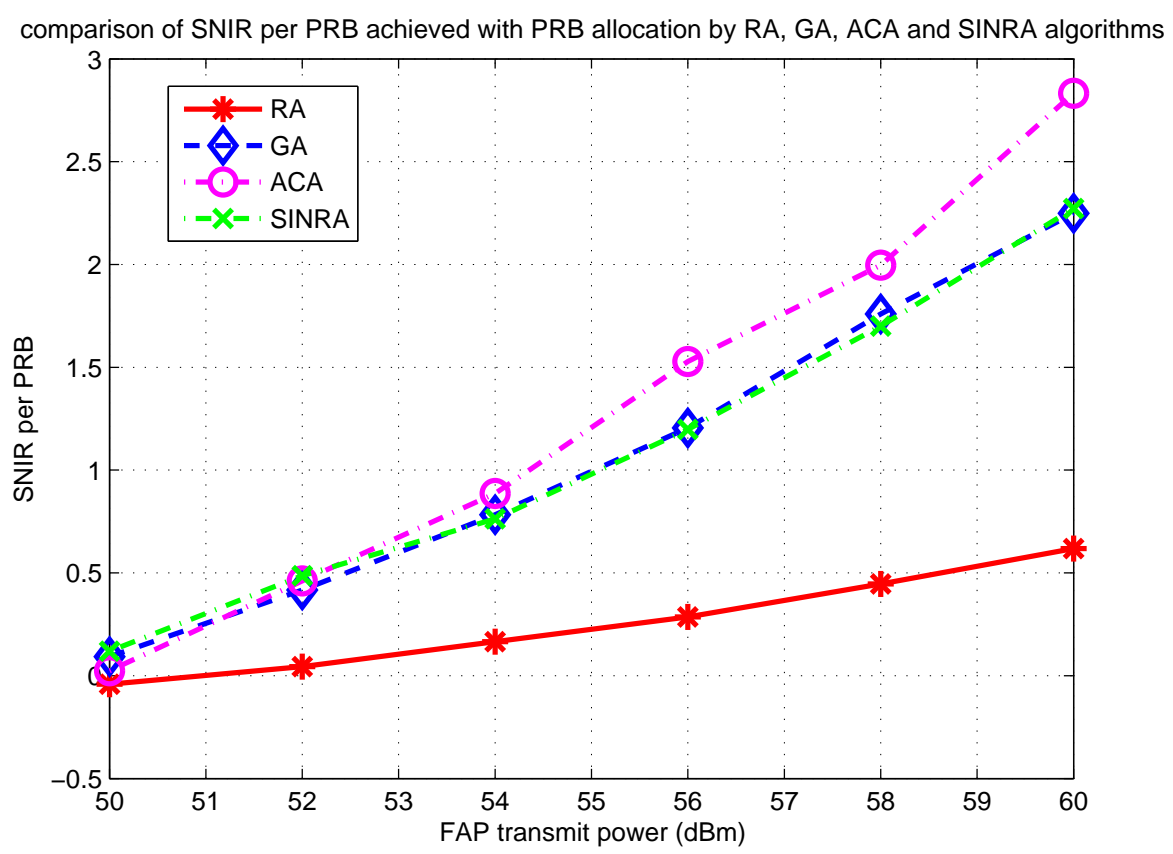

Figure 5.11: SINR per PRB vs. FAP Tx power Comparison of four algorithms

The graph of Figure 5.11 plots SINR per PRB performance with variation in FAP transmit power ranging from $50 \mathrm{dBm}$ to $60 \mathrm{dBm}$. The SINR per PRB performance improves with increase in FAP transmit power. The proposed algorithms outperform RA algorithm among which ACA exhibits the best SINR per PRB performance.

From Figure 5.12, we can conclude that ACA algorithm exhibits the best coverage per PRB performance compared to rest of the considered algorithms. Even GA and SINRA exhibit 30\% higher coverage per PRB performance compared to RA algorithm. 


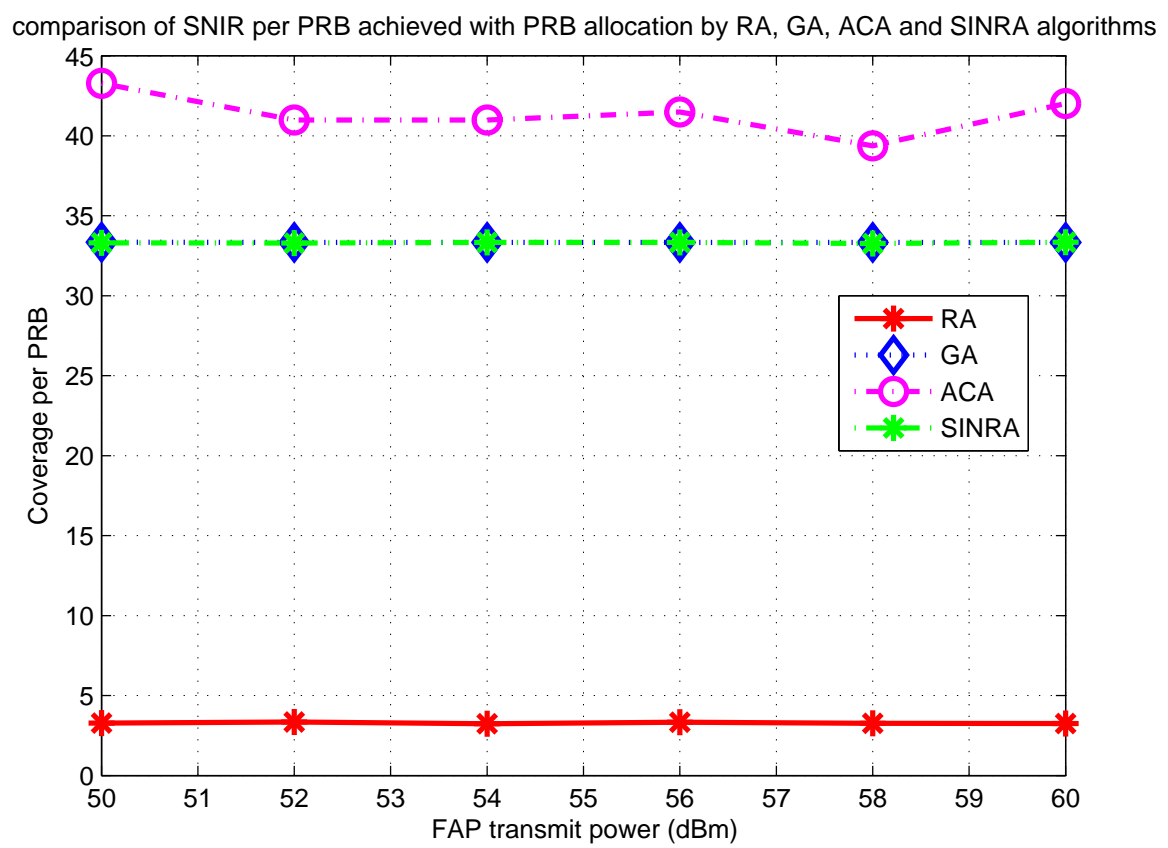

Figure 5.12: Coverage per PRB vs. FAP Tx power Comparison of four algorithms

\subsection{Summary}

The detailed performance analysis of proposed algorithms can be summarised as follows. Convergence analysis of evolutionary ACA algorithm, concludes that the algorithm converges after 12 iterations and therefore the ACA algorithm has been executed for 15 iterations while plotting rest of the performance graphs.

The comparative study of ACA, GA, SINRA and RA algorithms have been conducted in two separate subsections. In one of them the performance was analysed with the change in SINR threshold value $\left(\gamma_{t h}\right)$ while holding other system parameters constant at the suitable values. SINR performances of GA and SINR algorithms are better compared to ACA algorithm for lower values of $\gamma_{t h}$, i.e., for the PRB allocation problems with lower complexity. While ACA algorithm provides better and consistent SINR performance with increasing complexity of PRB allocation problem. GA and SINR algorithms provide very low outage probability while with RA algorithm implementation outage probability is very high i.e. with $\gamma_{t h}=5 \mathrm{~dB}$ results into complete outage. ACA algorithm provides near zero outage probabil- 


\begin{tabular}{|c|c|c|c|c|}
\hline Allocation algorithm & SINR & Outage probability & No of PRBs & Complexity \\
\hline \hline ACA & H & L & L & H \\
\hline SINRA & M & M & M & M \\
\hline GA & M & L & M & M \\
\hline RA & L & H & H & L \\
\hline
\end{tabular}

Table 5.1: Summary of performance analysis of proposed algorithm

ity irrespective of the value of $\gamma_{t h}$. Moreover, ACA algorithm provides efficient allocation of PRBs exhibiting better SINR and near zero outage probability with less number of PRBs utilised.

From the concluding graphs of Figure 5.5 and Figure 5.6, we can state that ACA outperforms the other algorithms as far as percentage of coverage per PRB and SINR per PRB parameters are concerned. Although considering the same parameters, SINRA and GA algorithms provide lower performance compared to ACA algorithm but their performance is an significant improvement over RA algorithm.

Similar conclusions can be drawn from graphs in the section where performance has been analyzed by varying FAP transmit power. In addition to this, it can be observed that the SINR performance of proposed algorithm improves with a raise in FAP transmit power. However, the outage probability and the number of required PRBs are insensitive to the variation in FAP transmit power. The results of comparative study of all proposed algorithms and random allocation algorithm can be summarized in Table 5.1, where, $\mathrm{H}$ means high performance, M means medium performance and L means low performance. From the Table 5.1, we can conclude that the ACA algorithm outperforms the other algorithms while GA and SINRA provide performance with the significant improvement over RA algorithm as far as the performance parameters such as average SINR experienced by network users, outage probability and number of required PRBs are concerned. The performance enhancement with ACA algorithm is at the expense of increased complexity of the algorithm which may lead to an inefficient usage of computational resources. 


\section{Chapter 6}

\section{Conclusion and Future work}

\subsection{Conclusion}

This thesis began with the study of LTE-A wireless communication standard. A forth coming LTE-A also known as $4 \mathrm{G}$ standard is the most promising wireless communication standard which supports higher peak rates, higher throughout, extended coverage and lower latencies. HetNet is one of the techniques adopted by LTE-A standard in order to provide higher data rate and extended indoor coverage. Among the challenges faced by HetNet or SCN technology, the challenge of interference mitigation due to shared spectrum between macro and femtocells is the prominent one. We chose to mitigate interference in LTE-A HetNet with intelligent sub-channel allocation to network users.

WE have presented sub-channel allocation problem as a graph coloring problem. With the goal of interference mitigation, we have proposed two PRB allocation algorithms namely greedy allocation and SINR based allocation which are based on the "Greedy algorithm for graph coloring problem". Further we have mathematically modelled the sub-channel allocation problem as an assignment type problem and proposed ant colony allocation based on ant colony optimization technique.

We have described the procedural details of the proposed algorithms and performed 
comparative performance analysis of these algorithms with each other and with random allocation algorithm. For the simulation purposes we have defined a HetNet network model following LTE-A standard. As well we have conducted sensitivity study of network model in order to validate it and to study an impact of system parameters one the network performance and hence on the performance of sub-channel allocation algorithm. The performance parameters such as an average SINR experienced by all network users, outage probability and the number of PRBs required for solution implementation were considered. From the conducted performance analysis we can conclude that, among all proposed algorithms ACA algorithm outperforms GA and SINR algorithms at the expense of increased complexity. Moreover, GA and SINRA algorithms provide a significant improvement over RA algorithm.

\subsection{Future work}

Although, ACA algorithm has been proven to outperform other proposed algorithms, it is complex in nature. The complexity of ACA algorithm leads to higher requirement of computational resources for the deployment of the algorithm. Further research could be conducted on the procedural details of ACA algorithm with the aim of improving efficiency of computational resource usage including execution time. 


\section{Bibliography}

[1] "LTE-Advanced." http://www.3gpp.org/lte-advanced. Accessed: 11/06/2012.

[2] S. Parkvall, E. Dahlman, A. Furuskar, Y. Jading, M. Olsson, S. Wanstedt, and K. Zangi, "LTE-Advanced - evolving lte towards IMT-Advanced," in Vehicular Technology Conference, 2008. VTC 2008-Fall. IEEE 68th, pp. 1 -5, Sept. 2008.

[3] C. S. Chen, F. Baccelli, and L. Roullet, "Joint optimization of radio resources in small and macro cell networks," in IEEE 73rd Vehicular Technology Conference (VTC Spring), (Budapest, Hungary), pp. 1-5, May 2011.

[4] T. Bäck, Evolutionary algorithms in theory and practice evolution strategies, evolutionary programming, genetic algorithms. Oxford, UK: Oxford University Press, 1996.

[5] H. Sandalidis, P. Stavroulakis, and J. Rodriguez-Tellez, "An efficient evolutionary algorithm for channel resource management in cellular mobile systems," Evolutionary Computation, IEEE Transactions on, vol. 2, pp. 125-137, Nov 1998.

[6] W. Hale, "Frequency assignment: Theory and applications," Proceedings of the IEEE, vol. 68, pp. 1497 - 1514, dec. 1980.

[7] D. Tissainayagam, D. Everitt, and M. Palaniswami, "A neural network driven solution to a channel assignment problem in wireless telephony," in International Conference on Neural Networks, vol. 6, pp. 133-137, Jun 1997. 
[8] S. Terrill, D. Everitt, and M. Palaniswami, "Neural networks for limited channel rearrangement in cellular mobile communication systems," in Neural Networks, 1994. IEEE World Congress on Computational Intelligence., 1994 IEEE International Conference on, vol. 6, pp. 3592 -3596 vol.6, jun- 2 jul 1994.

[9] N. Funabiki, N. Okutani, and S. Nishikawa, "A three-stage heuristic combined neuralnetwork algorithm for channel assignment in cellular mobile systems," Vehicular Technology, IEEE Transactions on, vol. 49, pp. 397 -403, Mar 2000.

[10] Z. He, Y. Zhang, C. Wei, and J. Wang, "A multistage self-organizing algorithm combined transiently chaotic neural network for cellular channel assignment," Vehicular Technology, IEEE Transactions on, vol. 51, pp. 1386 - 1396, Nov 2002.

[11] D. Beckmann and U. Killat, "A new strategy for the application of genetic algorithms to the channel-assignment problem," IEEE Transactions on Vehicular Technology, vol. 48, pp. $1261-1269$, July 1999.

[12] X. Fu, Y. Pan, A. Bourgeois, and P. Fan, "A three-stage heuristic combined genetic algorithm strategy to the channel-assignment problem," in Parallel and Distributed Processing Symposium, (Nice, France), p. 8, April 2003.

[13] J. Janssen and K. Kilakos, "An optimal solution to the ldquo;philadelphia rdquo; channel assignment problem," IEEE Transactions on Vehicular Technology, vol. 48, pp. 1012 -1014 , may 1999.

[14] S. Ghosh, B. Sinha, and N. Das, "Channel assignment using genetic algorithm based on geometric symmetry," IEEE Transactions on Vehicular Technology,, vol. 52, pp. 860 - 875, July 2003.

[15] R. Mathar and J. Mattfeldt, "Channel assignment in cellular radio networks," IEEE Transactions on Vehicular Technology, vol. 42, pp. 647 -656, Nov 1993. 
[16] M. Duque-Anton, D. Kunz, and B. Ruber, "Channel assignment for cellular radio using simulated annealing," IEEE Transactions on Vehicular Technology, vol. 42, pp. 14-21, Feb 1993.

[17] R. Montemanni, D. Smith, and S. Allen, "An ants algorithm for the minimum-span frequency-assignment problem with multiple interference," IEEE Transactions on Vehicular Technology, vol. 51, pp. 949 - 953, Sep 2002.

[18] R. Lin, K. Niu, W. Xu, and Z. He, "A two-level distributed sub-carrier allocation algorithm based on ant colony optimization in ofdma systems," in IEEE 71st Vehicular Technology Conference (VTC 2010-Spring), (Taipei, Taiwan), May 2010.

[19] H. Ahmadi, Y. H. Chew, and C. C. Chai, "Multicell multiuser ofdma dynamic resource allocation using ant colony optimization," in Vehicular Technology Conference (VTC Spring), (Budapest, Hungary), pp. 1 -5, May 2011.

[20] M. Dorigo, V. Maniezzo, and A. Colorni, "Ant system: optimization by a colony of cooperating agents," IEEE Transactions on Systems, Man, and Cybernetics, vol. 26, pp. $29-41$, Feb 1996.

[21] R. Madan, A. Sampath, A. Khandekar, J. Borran, and N. Bhushan, "Distributed interference management and scheduling in lte-a femto networks," in IEEE Global Telecommunications Conference (GLOBECOM 2010), (Florida USA), pp. 1-5, Dec. 2010.

[22] Y.-J. Chang, Z. Tao, J. Zhang, and C.-C. Kuo, "A graph-based approach to multicell ofdma downlink resource allocation," in Global Telecommunications Conference (GLOBECOM 2008), (New Orleans, USA), Dec 2008.

[23] K. Zheng, F. Hu, L. Lei, and W. Wang, "Interference coordination between femtocells in lte-advanced networks with carrier aggregation," in 5th International ICST Conference on Communications and Networking in China (CHINACOM), (Kunming, China), pp. 1 -5 , Aug. 2010. 
[24] G. Audhya, K. Sinha, K. Mandal, R. Dattagupta, S. Ghosh, and B. Sinha, "A new approach to fast near-optimal channel assignment in cellular mobile networks," Mobile Computing, IEEE Transactions on, vol. PP, no. 99, p. 1, 2012.

[25] A. Dalal, H. Li, and D. Agrawal, "Fractional frequency reuse to mitigate interference in self-configuring lte-femtocells network," in IEEE 8th International Conference on Mobile Adhoc and Sensor Systems (MASS), (Valencia, Spain), pp. 49 -54, Oct. 2011.

[26] V. Chandrasekhar, J. Andrews, Z. Shen, T. Muharemovic, and A. Gatherer, "Distributed power control in femtocell-underlay cellular networks," in Global Telecommunications Conference IEEE, pp. 1 -6, Dec 2009.

[27] M. Maso, L. Cardoso, M. Debbah, and L. Vangelista, "Channel estimation impact for lte small cells based on mu-vfdm," in IEEE Wireless Communications and Networking Conference (WCNC), (Shanghai, China), pp. 2560 -2565, April 2012.

[28] C. de Lima, M. Bennis, and M. Latva-aho, "Coordination mechanisms for self-organizing femtocells in two-tier coexistence scenarios," IEEE Transactions on Wireless Communications, vol. 11, pp. 2212 -2223, June 2012.

[29] S. ping Yeh, S. Talwar, N. Himayat, and K. Johnsson, "Power control based interference mitigation in multi-tier networks," in IEEE GLOBECOM Workshops, (Florida, USA), pp. $701-705$, Dec. 2010.

[30] K. Balachandran, J. Kang, K. Karakayali, and K. Rege, "Cell selection with downlink resource partitioning in heterogeneous networks," in IEEE International Conference on Communications(ICC), (Ottawa, Canada), June 2011.

[31] S. Akoum, M. Zwingelstein-Colin, R. Heath, and M. Debbah, "Cognitive cooperation for the downlink of frequency reuse small cells," in 2nd International Workshop on Cognitive Information Processing (CIP), pp. 111 -115, June 2010. 
[32] J. Riihijarvi, M. Petrova, and P. Mahonen, "Frequency allocation for wlans using graph colouring techniques," in Second Annual Conference on Wireless On-demand Network Systems and Services (WONS), pp. 216 - 222, Jan. 2005.

[33] "An overview of the LTE physical layer part I." http://www.3gpp.org/ftp/workshop/2009-12-17_ITU-R_IMT-Adv_eval/docs/pdf/REV-09000 Accessed: 11/06/2012.

[34] L. Tan, Z. Feng, W. Li, Z. Jing, and T. A. Gulliver, "Graph coloring based spectrum allocation for femtocell downlink interference mitigation," in Wireless Communications and Networking Conference (WCNC), 2011 IEEE, vol. 3, p. 1248, March 2011.

[35] R. Madan, J. Borran, A. Sampath, N. Bhushan, A. Khandekar, and T. Ji, "Cell association and interference coordination in heterogeneous lte-a cellular networks," IEEE Journal on Selected Areas in Communications, vol. 28, pp. 1479 -1489, Dec 2010.

[36] D. Costa and A. Hertz, "Ants Can Colour Graphs," Journal of the operational research society, vol. 48, no. 3, pp. 295-305, 1997.

[37] A. P. Engelbrecht, Computational Intelligence: An Introduction. Wiley Publishing, 2nd ed., 2007.

[38] M. Dorigo, M. Birattari, and T. Stutzle, "Ant colony optimization," IEEE Computational Intelligence Magazine, vol. 1, pp. 28 -39, nov. 2006.

[39] D. Brélaz, "New methods to color the vertices of a graph," Commun. ACM, vol. 22, pp. 251-256, Apr 1979.

[40] T. H. Cormen, C. E. Leiserson, R. L. Rivest, and C. Stein, Introduction to Algorithms, Third Edition. The MIT Press, 3rd ed., 2009.

[41] J. Hamblin, Math for Liberal Studies. CreateSpace, 2nd ed., 2012. 